\title{
MAXIMAL $R$-SETS, GRASSMANN SPACES, AND STIEFEL SPACES OF A HILBERT SPACE
}

\author{
BY \\ ERHARD LUFT
}

1. Introduction. The ordinary Fredholm theory of Hilbert spaces was generalized in [4] and [5] to apply to a wider range of problems in analysis. The ideal of compact linear maps, which plays a fundamental role in the ordinary theory, was replaced by a certain class of $C^{*}$-algebras called $R$-algebras in [4]. This generalization is based on the Rellich criterion for compact linear maps of a Hilbert space, which states that a linear map is compact if and only if for every $\varepsilon>0$ there exists a closed linear subspace of finite codimension such that the norm of the restriction of the linear map to this subspace becomes smaller than $\varepsilon$. The $R$-algebras in [4] are defined by substituting for the collection of closed linear subspaces of finite codimension in this criterion a collection of closed linear subspaces which is an $R$-set.

The $R$-sets are subsets of the metric space of all closed linear subspaces of a Hilbert space, which satisfy a condition related to the metric. For the explicit definition we refer to Chapter 4 . Each $R$-set is contained in a uniquely determined smallest maximal $R$-set. The algebraic objects attached to an $R$-set depend on this maximal $R$-set only, and its structure is of crucial importance.

The path components of a maximal $R$-set are simply Grassmann manifolds for a finite-dimensional Hilbert space. In the following we present a general theory of Grassmann and Stiefel manifolds associated with a maximal $R$-set of a Hilbert space. This theory contains many important features of the ordinary theory of Grassmann and Stiefel manifolds of a finite-dimensional Hilbert space. Our constructions and arguments are independent of the dimension, and the ordinary theory for finite-dimensional Hilbert spaces and its direct generalization to Hilbert spaces of arbitrary dimension appear as special cases.

We show that the path components of a maximal $R$-set are Banach manifolds and homogeneous spaces determined by the action of the group of continuous isomorphisms of the Hilbert space onto itself which leave the maximal $R$-set invariant. Furthermore, there is a natural fibre bundle structure.

Several other Banach manifolds are associated in a canonical way with a path component of a maximal $R$-set. They are homogeneous spaces with a fibre bundle structure and direct analogues of the ordinary Stiefel manifolds.

Finally, we investigate the maximal $R$-set of all closed linear subspaces of a

Received by the editors December 20, 1965. 
Hilbert space. This special case gives directly the ordinary theory of Grassmann and Stiefel spaces for arbitrary not necessarily finite-dimensional Hilbert spaces. The result of [9], that the general linear group of an infinite-dimensional Hilbert space is contractible, implies the Grassmann and Stiefel spaces of an infinitedimensional Hilbert space are universal classifying spaces for the general linear group of certain Hilbert spaces.

We tried to make this manuscript as self-contained as possible. We avoided any spectral theory and elaborated a purely "geometric" approach. Further applications are planned in subsequent publications.

I would like to express my great appreciation to Professor H. O. Cordes for many helpful and inspiring conversations and for his interest in this work. Much of Chapters 3, 4, and 5 is elaborated from his papers [4] and [5]. I would also like to thank the referee for a very useful suggestion. And I would like to thank Professor D. Bures for a useful conversation.

2. Preliminaries. $H$ denotes in the following a real, complex, or quaternionic Hilbert space, which also can be finite dimensional. If $x, y \in H$, then $(x, y)$ denotes the inner product of $x$ and $y$, and $\|x\|=(x, x)^{1 / 2}$ the length of $x$. If $H$ and $K$ are two Hilbert spaces, then $\mathfrak{L}(H, K)$ is the linear space of continuous (=bounded) linear maps (=operators) from $H$ to $K$ with the norm

$$
\|\alpha\|=\sup \{\|\alpha(x)\| ; x \in H \text { and }\|x\|=1\} \text { for each } \alpha \in \mathfrak{L}(H) \text {. }
$$

We denote $\mathfrak{L}(H, H)$ by $\mathfrak{L}(H) .1 \in \mathfrak{L}(H)$ is the identity map. The general linear group $\mathrm{GL}(H)$ of the Hilbert space $H$ is the topological subspace of $\mathfrak{L}(H)$ consisting of the invertible elements of $\mathfrak{L}(H)$. It is an open subset of $\mathfrak{L}(H)$. The mapping Inv: $\mathrm{GL}(H) \rightarrow \mathrm{GL}(H)$ which assigns to any $\gamma \in \mathrm{GL}(H)$ its inverse $\operatorname{Inv}(\gamma)=\gamma^{-1}$ is continuous. Consequently, $\mathrm{GL}(H)$ is a topological group. An element $\mu \in \mathrm{GL}(H)$ is called unitary or orthogonal if $\|\mu(x)\|=\|x\|$ for all $x \in H$. The unitary elements of $\mathrm{GL}(H)$ form a closed subgroup $\mathrm{U}(H)$ of $\mathrm{GL}(H)$.

If $S$ is a closed linear subspace, then $S^{\perp}$ denotes the orthogonal complement of $S$ in $H$, and $\pi_{S}: H \rightarrow H$ the orthogonal projection of $H$ onto $S$. We notice that $\pi_{S} \in \mathfrak{L}(H),\left\|\pi_{S}\right\|=1$, and $\pi_{S^{\perp}}=\mathbf{1}-\pi_{S}$.

THEOREM 2.1. If $\gamma \in \mathfrak{L}(H, K)$ maps $H$ one-to-one onto $K$ then $\gamma^{-1} \in \mathfrak{Q}(K, H)$.

Proof. See, for example, [11, p. 18].

For each $\alpha \in \mathfrak{L}(H, K)$ the adjoint $\alpha^{*} \in \mathfrak{L}(K, H)$ is defined. A map $\alpha \in \mathfrak{L}(H)$ is called self-adjoint, if $\alpha^{*}=\alpha$. We recall a few elementary properties of the adjoint operation which will be frequently used.

LEMMA 2.1. $(\alpha(x), y)=\left(x, \alpha^{*}(y)\right)$ for each $x \in H, y \in K$ and $\alpha \in \mathfrak{L}(H, K)$ (definition). If $\alpha \in \mathfrak{Q}(H, K)$, then $\left\|\alpha^{*}\right\|=\|\alpha\|$, kernel $(\alpha)=\left(\alpha^{*}(K)\right)^{\perp}$, kernel $\left(\alpha^{*}\right)=(\alpha(H))^{\perp}$, and $\alpha(H)$ is closed if and only if $\alpha^{*}(K)$ is closed. If $\alpha \in \mathfrak{L}(H, K)$ and $\beta \in \mathfrak{L}(K, L)$, then 
$(\beta \cdot \alpha)^{*}=\alpha^{*} \cdot \beta^{*}$. The orthogonal projection $\pi_{S}$ of the Hilbert space $H$ onto the closed linear subspace $S$ of $H$ is self-adjoint.

LEMma 2.2. If $\alpha \in \mathfrak{L}(H)$ and $S$ is a closed linear subspace with $\alpha(S)$ is closed, then there exists $a \beta \in \mathfrak{Q}(H)$ such that

$$
\pi_{\alpha(S)}=\alpha \cdot \pi_{S} \cdot \beta \cdot \pi_{S} \cdot \alpha^{*} .
$$

Proof. We consider the map $\tilde{\alpha}=\alpha \cdot \pi_{S}: H \rightarrow H$. We have $\operatorname{kernel}(\tilde{\alpha})=\left(\tilde{\alpha}^{*}(H)\right)^{\perp}$ and $\operatorname{kernel}\left(\tilde{\alpha}^{*}\right)=(\tilde{\alpha}(H))^{\perp}$. Since $\tilde{\alpha}(H)=\alpha(S)$ is closed, we conclude that $\left(\operatorname{kernel}\left(\tilde{\alpha}^{*}\right)\right)^{\perp}$ $=\alpha(S)$ and $(\operatorname{kernel}(\tilde{\alpha}))^{\perp}=\tilde{\alpha}^{*}(H)$. The restrictions $\tilde{\alpha}^{\prime}: \tilde{\alpha}^{*}(H) \rightarrow \alpha(S)$ of $\tilde{\alpha}$ and $\tilde{\alpha}^{* \prime}: \alpha(S) \rightarrow \tilde{\alpha}^{*}(H)$ of $\tilde{\alpha}^{*}$ are therefore well defined and isomorphisms by Theorem 2.1. We observe that $\tilde{\alpha}^{*}=\tilde{\alpha}^{*} \cdot \pi_{\alpha(S)}$. Consider $\left(\tilde{\alpha}^{\prime}\right)^{-1} \cdot\left(\tilde{\alpha}^{* \prime}\right)^{-1}: \tilde{\alpha}^{*}(H) \rightarrow \tilde{\alpha}^{*}(H)$, and let $\beta=\iota \cdot\left(\left(\tilde{\alpha}^{\prime}\right)^{-1} \cdot\left(\tilde{\alpha}^{* \prime}\right)^{-1}\right) \cdot \pi \in \mathfrak{L}(H)$, where $\iota: \tilde{\alpha}^{*}(H) \rightarrow H$ is the natural inclusion and $\pi: H \rightarrow \tilde{\alpha}^{*}(H)$ is the orthogonal projection onto the closed linear subspace $\tilde{\alpha}^{*}(H)$. From the construction follows immediately

$$
\pi_{\alpha(S)}=\tilde{\alpha} \cdot \beta \cdot \tilde{\alpha}^{*} \cdot \pi_{\alpha(S)}=\tilde{\alpha} \cdot \beta \cdot \tilde{\alpha}^{*}=\alpha \cdot \pi_{S} \cdot \beta \cdot \pi_{S} \cdot \alpha^{*} .
$$

The following definition will be very useful.

Definition 2.1. Let $\alpha \in \mathfrak{L}(H, K)$ and let $S$ be a linear subspace of $H$, then

$$
c(S, \alpha)=\inf \{\|\alpha(x)\| ; x \in S \text { and }\|x\|=1\} .
$$

Lemma 2.3. Let $\alpha \in \mathfrak{L}(H, K)$ and let $S$ be a closed linear subspace of $H$. Then kernel $(\alpha) \cap S=\{o\}$, and $\alpha(S)$ is closed if and only if $c(S, \alpha)>0$. If $c(S, \alpha)>0$, then the restriction $\alpha^{\prime}: S \rightarrow \alpha(S)$ is an isomorphism and

$$
c(S, \alpha)=\left(\left\|\alpha^{\prime-1}\right\|\right)^{-1} .
$$

Proof. Suppose $c(S, \alpha)>0$. Then certainly kernel $(\alpha) \cap S=\{o\}$. Now let $y \in K$ and let $\left\{\alpha\left(x_{n}\right)\right\}_{n=1}^{\infty}$ be a sequence with $\lim \alpha\left(x_{n}\right)=y$. The sequence $\left\{x_{n}\right\}_{n=1}^{\infty}$ is then a Cauchy sequence since

$$
\left\|x_{n}-x_{m}\right\| \leqq(c(S, \alpha))^{-1} \cdot\left\|\alpha\left(x_{n}\right)-\alpha\left(x_{m}\right)\right\| .
$$

Let $x=\lim x_{n}$. Then $\alpha(x)=y$. Therefore $\alpha(S)$ is closed. Assume now kernel $(\alpha) \cap S$ $=\{o\}$ and $\alpha(S)$ is closed. $\alpha(S)$ is a Hilbert space and we consider the restriction $\alpha^{\prime}: S \rightarrow \alpha(S)$. By Theorem 2.1, $\alpha^{\prime-1} \in \mathfrak{R}(\alpha(S), S)$. We have

$$
\frac{\left\|\alpha^{\prime-1}\left(\alpha^{\prime}(x)\right)\right\|}{\left\|\alpha^{\prime}(x)\right\|}=\left(\frac{\left\|\alpha^{\prime}(x)\right\|}{\|x\|}\right)^{-1} \leqq(c(S, \alpha))^{-1} \text { for } x \in S \text { and } x \neq o .
$$

With the first expression we can approximate $\left\|\alpha^{\prime-1}\right\|$.

Lemma 2.4. Suppose $\alpha \in \mathfrak{L}(H, K)$ with $\alpha(H)$ is closed. Then we have

$$
c\left(\alpha(H), \alpha^{*}\right)=c\left(\alpha^{*}(H), \alpha\right) .
$$


Proof. Since $\alpha(H)$ is closed, $\alpha^{*}(K)$ is closed also (Lemma 2.1). The restrictions $\alpha^{\prime}: \alpha^{*}(K) \rightarrow \alpha(H)$ of $\alpha$ and $\alpha^{*^{\prime}}: \alpha(H) \rightarrow \alpha^{*}(K)$ of $\alpha^{*}$ are well defined and isomorphisms (Theorem 2.1).

$$
\begin{aligned}
c\left(\alpha(H), \alpha^{*}\right) & =\left(\left\|\alpha^{*-1}\right\|\right)^{-1}=\left(\left\|\alpha^{*-1}\right\|\right)^{-1}=\left(\left\|\left(\alpha^{\prime-1}\right)^{*}\right\|\right)^{-1}=\left(\left\|\alpha^{\prime-1}\right\|\right)^{-1} \\
& =c\left(\alpha^{*}(H), \alpha\right) .
\end{aligned}
$$

Definition 2.2. $\mathfrak{A} \subset \mathfrak{L}(H)$ is called a $C^{*}$-algebra, if $\mathfrak{A}$ is a subalgebra of $\mathfrak{L}(H)$, a closed subset of $\mathfrak{Q}(H)$, and $(\mathfrak{A})^{*}=\mathfrak{A}$. If $1 \in \mathfrak{A}$, then we have the closed subgroups

$$
\begin{aligned}
\mathrm{GL}(\mathfrak{A}) & =\left\{\gamma ; \gamma \in \mathrm{GL}(H) \text { with } \gamma, \gamma^{-1} \in \mathfrak{A}\right\} \text { and } \\
\mathrm{U}(\mathfrak{A}) & =\mathrm{U}(H) \cap \mathfrak{A}
\end{aligned}
$$

of the topological group $\mathrm{GL}(H)$.

$\mathrm{GL}(\mathfrak{U})$ is an open subset of the Banach space $\mathfrak{A}$. (There is an open neighborhood of 1 in $\mathfrak{A}$ contained in GL( $\mathfrak{X})$, since for $\gamma$ sufficiently close to 1 the inverse exists and can be represented by a geometric series which is convergent in $\mathfrak{A}$.)

Lemma 2.5. Let $\mathfrak{A} \subset \mathfrak{Q}(H)$ be a $C^{*}$-algebra with $1 \in \mathfrak{A}$. If $\gamma \in \mathrm{GL}(H) \cap \mathfrak{A}$ is selfadjoint, then $\gamma^{-1} \in \mathrm{GL}(H) \cap \mathfrak{A}$.

Proof. Consider $c \cdot \gamma^{2}$, where $c$ is a real number. We compute

$$
\begin{aligned}
&\left(\left(c \cdot \gamma^{2}-1\right)(x),\left(c \cdot \gamma^{2}-1\right)(x)\right)=c^{2} \cdot\left(\gamma^{2}(x), \gamma^{2}(x)\right)-2 c \cdot(\gamma(x), \gamma(x))+(x, x) \\
& \leqq c^{2} \cdot\|\gamma\|^{4} \cdot\|x\|^{2}-2 c \cdot\left(\left\|\gamma^{-1}\right\|\right)^{-2} \cdot\|x\|^{2}+\|x\|^{2} \\
&=\left[1+\|\gamma\|^{4} \cdot\left(c-\left(\|\gamma\|^{2} \cdot\left\|\gamma^{-1}\right\|\right)^{-2}\right)^{2}-\left(\|\gamma\| \cdot\left\|\gamma^{-1}\right\|\right)^{-4}\right] \cdot\|x\|^{2} .
\end{aligned}
$$

We conclude that there exists a $c \neq 0$ such that $\left\|c \cdot \gamma^{2}-1\right\|<1$. Since we have $c \cdot \gamma^{2}=1+\left(c \cdot \gamma^{2}-1\right)$ and $\left\|c \cdot \gamma^{2}-1\right\|<1$, the geometric series $\sum_{n=0}^{\infty}(-1)^{n} \cdot\left(c \cdot \gamma^{2}-1\right)^{n}$ is convergent in $\mathfrak{A}$ and gives $\left(c \cdot \gamma^{2}\right)^{-1} \in \mathfrak{A}$. Then also $\left(\gamma^{2}\right)^{-1} \in \mathfrak{A}$ and $\gamma^{-1}$ $=\gamma \cdot\left(\gamma^{2}\right)^{-1} \in \mathfrak{A}$.

LEMMA 2.6. Recall that a self-adjoint $\alpha \in \mathfrak{Q}(H)$ is said to be positive, if $(\alpha(x), x) \geqq O$ for all $x \in H$. Let $\mathfrak{A} \subset \mathfrak{L}(H)$ be a $C^{*}$-algebra with $1 \in \mathfrak{A}$. Suppose $\alpha \in \mathfrak{A}$ is self-adjoint and positive. Then there exists a unique self-adjoint and positive $\beta \in \mathfrak{A}$ with $\beta^{2}=\alpha$. If further $S \subset H$ is a closed linear subspace with $\alpha(S) \subset S$, then also $\beta(S) \subset S$, and if $\left.\alpha\right|_{S}=i d$, then also $\left.\beta\right|_{S}=i d$.

Proof. We may assume that $1-\alpha$ is positive. We consider the sequence $\left\{\beta_{n}\right\}_{n=0}^{\infty}$ in $\mathfrak{A}$ defined by $\beta_{n+1}=\beta_{n}+\frac{1}{2}\left(\alpha-\beta_{n}^{2}\right)$ and $\beta_{0}=o$. This sequence is convergent. Let $\beta=\lim \beta_{n} \in \mathfrak{A}$. Then $\beta$ has the desired properties. See, for example, [12, p. 15].

Lemma 2.7. Suppose $\alpha \in \mathfrak{L}(H)$ is self-adjoint and positive. Then $1+\alpha \in \mathrm{GL}(H)$ and $\left\|(1+\alpha)^{-1}\right\| \leqq 1$. 
Proof. By Lemma 2.6 there is a $\beta \in \mathfrak{L}(H)$ with $\beta^{*}=\beta$ and $\beta^{2}=\alpha$. We compute for $x \in H$

$$
\|(1+\alpha)(x)\|^{2}=(x, x)+2 \cdot(\beta(x), \beta(x))+(\alpha(x), \alpha(x)) \geqq\|x\|^{2} .
$$

This implies $c(H,(1+\alpha)) \geqq 1$. Therefore $1+\alpha$ is injective and $(1+\alpha)(H)$ is closed (Lemma 2.3). Since $(\mathbf{1}+\alpha)^{*}=\mathbf{1}+\alpha$, it follows that $\mathbf{1}+\alpha$ is also surjective (Lemma 2.1). By Theorem 2.1 then $1+\alpha \in \mathrm{GL}(H)$. Finally, by Lemma 2.3

$$
\left\|(1+\alpha)^{-1}\right\|=(c(H,(1+\alpha)))^{-1} \leqq 1 .
$$

TheOREM 2.2. Let $\mathfrak{A} \subset \mathfrak{Q}(H)$ be a $C^{*}$-algebra with $1 \in \mathfrak{A}$. For each $\gamma \in \mathrm{GL}(H) \cap \mathfrak{A}$ there is a unique decomposition $\gamma=\mu \cdot \kappa$ such that $\mu \in \mathrm{U}(H) \cap \mathfrak{A}$ and $\kappa \in \mathrm{GL}(H) \cap \mathfrak{A}$ is self-adjoint and positive. The map

$$
\begin{aligned}
& u: \mathrm{GL}(H) \cap \mathfrak{A} \rightarrow \mathrm{U}(H) \cap \mathfrak{A}, \\
& u(\gamma)=\mu,
\end{aligned}
$$

is continuous.

Proof. Consider $\gamma^{*} \cdot \gamma \in \mathrm{GL}(H) \cap \mathfrak{A}$. We have $\gamma^{*} \cdot \gamma$ is self-adjoint and positive. By Lemma 2.6 there exists a positive self-adjoint $\beta \in \mathfrak{A}$ with $\beta^{2}=\gamma^{*} \cdot \gamma$. Obviously $\beta$ is injective and surjective since $\beta^{2} \in \mathrm{GL}(H)$. Therefore by Theorem 2.1 $\beta \in \mathrm{GL}(H) \cap \mathfrak{A}$, and by Lemma 2.5 also $\beta^{-1} \in \mathrm{GL}(H) \cap \mathfrak{A}$. Let $\kappa=\beta$ and $\mu=\gamma \cdot \beta^{-1}$. Then $\gamma=\mu \cdot \kappa$, and $\kappa$ is self-adjoint and positive. We compute $\mu^{*} \cdot \mu=\beta^{-1} \cdot \gamma^{*} \cdot \gamma \cdot \beta^{-1}=\beta^{-1} \cdot \beta^{2} \cdot \beta^{-1}=1$, i.e., $\mu \in \mathrm{U}(H) \cap \mathfrak{A}$.

To prove the uniqueness of the decomposition, we assume another representation $\gamma=\tilde{\mu} \cdot \tilde{\kappa}$ with the above properties. Then $\mu \cdot \kappa=\tilde{\mu} \cdot \tilde{\kappa}$, or $\kappa=\mu^{*} \cdot \tilde{\mu} \cdot \tilde{\kappa}$, and hence $\kappa=\kappa^{*}=\tilde{\kappa} \cdot \tilde{\mu}^{*} \cdot \mu$. It follows that $\kappa^{2}=\tilde{\kappa}^{2}=\gamma^{*} \cdot \gamma$. From the uniqueness of $\beta$ in Lemma 2.6, we conclude $\kappa=\tilde{\kappa}$ and $\mu=\tilde{\mu}$.

To prove the continuity of $u$, it is sufficient to show that the map

$$
w: \mathrm{GL}(H) \cap \mathfrak{A} \rightarrow \mathrm{GL}(H) \cap \mathfrak{A},
$$

$w(\gamma)=\beta$, where $\beta$ is the unique self-adjoint and positive element with $\beta^{2}=\gamma^{*} \cdot \gamma$, is continuous. Let $w\left(\gamma_{0}\right)=\beta_{0}, w(\gamma)=\beta$.

We apply to $\beta_{0}$ and $\beta$ Lemma 2.6 , and we obtain $\beta_{0}=\delta_{0}^{2}, \beta=\delta^{2}$ with $\delta_{0}^{*}=\delta_{0}$, $\delta^{*}=\delta$, and again $\delta_{0}, \delta \in \mathrm{GL}(H)$. Then we decompose

$$
\beta_{0}+\beta=\delta_{0} \cdot\left(1+\left(\delta_{0}^{-1} \cdot \delta\right) \cdot\left(\delta \cdot \delta_{0}^{-1}\right)\right) \cdot \delta_{0}
$$

Let $\varepsilon=\delta \cdot \delta_{0}^{-1}$. Now $\varepsilon^{*} \cdot \varepsilon$ is self-adjoint and positive. By Lemma 2.7 then $1+\varepsilon^{*} \cdot \varepsilon \in \mathrm{GL}(H)$ and $\left\|\left(1+\varepsilon^{*} \cdot \varepsilon\right)^{-1}\right\| \leqq 1$. We compute

$$
\begin{aligned}
\left\|\beta_{0}-\beta\right\| & =\left\|\left(\beta_{0}-\beta\right) \cdot\left(\beta_{0}+\beta\right) \cdot\left(\beta_{0}+\beta\right)^{-1}\right\| \leqq\left\|\beta_{0}^{2}-\beta^{2}\right\| \cdot\left\|\left(\beta_{0}+\beta\right)^{-1}\right\| \\
& =\left\|\gamma_{0}^{*} \cdot \gamma_{0}-\gamma^{*} \cdot \gamma\right\| \cdot\left\|\delta_{0}^{-1} \cdot\left(1+\varepsilon^{*} \cdot \varepsilon\right)^{-1} \cdot \delta_{0}^{-1}\right\| \\
& \leqq\left\|\gamma_{0}^{*} \cdot \gamma_{0}-\gamma^{*} \cdot \gamma\right\| \cdot\left\|\delta_{0}^{-1}\right\|^{2} .
\end{aligned}
$$

Which proves the continuity of $w$. 
CoRollaRy 2.1. Let $\mathfrak{A} \subset \mathfrak{Q}(H)$ be a $C^{*}$-algebra with $1 \in \mathfrak{A}$. If $\gamma \in \mathrm{GL}(H) \cap \mathfrak{A}$, then also $\gamma^{-1} \in \mathrm{GL}(H) \cap \mathfrak{A}$, i.e.,

$$
\mathrm{GL}(\mathfrak{A})=\mathrm{GL}(H) \cap \mathfrak{A} .
$$

Proof. By Theorem 2.2, $\gamma=\mu \cdot \kappa$ with $\mu \in \mathrm{U}(H) \cap \mathfrak{A}, \kappa \in \mathrm{GL}(H) \cap \mathfrak{A}$, and $\kappa$ is self-adjoint. Then $\gamma^{-1}=\kappa^{-1} \cdot \mu^{*}$. By Lemma $2.5, \kappa^{-1} \in \mathrm{GL}(H) \cap \mathfrak{A}$, which proves $\gamma^{-1} \in \mathrm{GL}(H) \cap \mathfrak{A}$.

CoRollary 2.2. Let $\mathfrak{A} \subset \mathfrak{Q}(H)$ be a $C^{*}$-algebra with $1 \in \mathfrak{A}$. For each $\gamma \in \mathrm{GL}(\mathfrak{X})$ there is a unique decomposition $\gamma=\mu \cdot \kappa$ such that $\mu \in \mathrm{U}(\mathfrak{X})$ and $\kappa \in \mathrm{GL}(\mathfrak{X})$ is selfadjoint and positive. The map

$$
\begin{aligned}
& u: \mathrm{GL}(\mathfrak{A}) \rightarrow \mathrm{U}(\mathfrak{A}), \\
& u(\gamma)=\mu,
\end{aligned}
$$

is continuous. We notice further the following property: Let $S \subset \mathrm{H}$ be a closed linear subspace. Suppose $\gamma \in \mathrm{GL}(\mathfrak{H})$ satisfies $\gamma\left(S^{\perp}\right)=(\gamma(S))^{\perp}$, then $u(\gamma)(S)=\gamma(S)$. And if $\gamma_{1}, \gamma_{2} \in \mathrm{GL}(\mathfrak{X})$ satisfy $\gamma_{i}\left(S^{\perp}\right)=\left(\gamma_{i}(S)\right)^{\perp}, i=1,2$, and $\left.\gamma_{1}\right|_{s}=\left.\gamma_{2}\right|_{s}$, then $\left.u\left(\gamma_{1}\right)\right|_{s}=\left.u\left(\gamma_{2}\right)\right|_{s}$.

Proof. Theorem 2.2 and Corollary 2.1 prove the first part of the corollary. $\gamma\left(S^{\perp}\right)=(\gamma(S))^{\perp}$ implies $\gamma^{*} \cdot \gamma(S)=S$ and $\gamma^{*} \cdot \gamma\left(S^{\perp}\right)=S^{\perp}$. The corresponding $\beta$ satisfies then also $\beta(S)=S$ and $\beta\left(S^{\perp}\right)=S^{\perp}$ (Lemma 2.6). Hence $u(\gamma)(S)=\mu(S)=\left(\gamma \cdot \beta^{-1}\right)(S)$ $=\gamma(S)$.

To prove the last property, observe again $\gamma_{i}^{*} \cdot \gamma_{i}(S)=S$ and $\gamma_{i}^{*} \cdot \gamma_{i}\left(S^{\perp}\right)=S^{\perp}$, $i=1,2$. We compute the corresponding $\beta_{i}$ by computing $\left.\beta_{i}\right|_{S}$ and $\left.\beta_{i}\right|_{S^{\perp}}$ separately and forming $\beta_{i}=\left.\left.\beta_{i}\right|_{s} \oplus \beta_{i}\right|_{S^{\perp}}, i=1,2$. But $\left.\gamma_{1}^{*} \cdot \gamma_{1}\right|_{s}=\left.\gamma_{2}^{*} \cdot \gamma_{2}\right|_{s}$, therefore $\left.\beta_{1}\right|_{s}=\left.\beta_{2}\right|_{s}$, and hence $\left.\mu_{1}\right|_{s}=\left.\mu_{2}\right|_{s}$.

THEOREM 2.3. Let $\mathfrak{A} \subset \mathfrak{Q}(H)$ be a $C^{*}$-algebra with $1 \in \mathfrak{A}$, and let $S \subset H$ be a closed linear subspace with $\pi_{S} \in \mathfrak{A}$. We consider the closed subgroups

$$
\begin{aligned}
\mathrm{GL}(\mathfrak{U})_{S} & =\{\gamma ; \gamma \in \mathrm{GL}(\mathfrak{U}) \text { with } \gamma(S)=S\}, \\
\mathrm{U}(\mathfrak{H})_{S} & =\mathrm{GL}(\mathfrak{H})_{S} \cap \mathrm{U}(H)
\end{aligned}
$$

of the topological groups $\mathrm{GL}(\mathfrak{H})$ and $\mathrm{U}(\mathfrak{A})$. Then $\mathrm{U}(\mathfrak{A})_{S} \subset \mathrm{GL}(\mathfrak{A})_{S}$ is a strong deformation retract of the space $\mathrm{GL}(\mathfrak{U})_{S}$. In particular $\mathrm{U}(\mathfrak{U})_{S}$ and $\mathrm{GL}(\mathfrak{U})_{S}$ are of the same homotopy type.

Proof. We introduce the closed subgroup

$$
\mathrm{GL}(\mathfrak{U})_{S, S^{\perp}}=\left\{\gamma ; \gamma \in \mathrm{GL}(\mathfrak{U}) \text { with } \gamma(S)=S \text { and } \gamma\left(S^{\perp}\right)=S^{\perp}\right\}
$$

of the group $\mathrm{GL}(\mathfrak{A})_{S}$. We have the strong deformation retract map

$$
\begin{aligned}
& r: \mathrm{GL}(\mathfrak{U})_{S} \rightarrow \mathrm{GL}(\mathfrak{U})_{S, S^{\perp}}, \\
& r(\gamma)=\gamma \cdot \pi_{S}+\pi_{S^{\perp}} \cdot \gamma \cdot \pi_{S^{\perp}}=\gamma-\pi_{S} \cdot \gamma \cdot \pi_{S^{\perp}} .
\end{aligned}
$$


(Certainly $r(\gamma)(S) \subset S$ and $r(\gamma)\left(S^{\perp}\right) \subset S^{\perp}$. We compute directly $r(\gamma) \cdot r\left(\gamma^{-1}\right)=1$ and $r\left(\gamma^{-1}\right) \cdot r(\gamma)=1$. Therefore $r(\gamma) \in \mathrm{GL}(\mathfrak{Q})_{S, S^{\perp}}$, and $r$ is well defined.)

A deformation retract homotopy is given by

$$
\begin{aligned}
& \hat{r}: \mathrm{GL}(\mathfrak{A})_{S} \times[0,1] \rightarrow \mathrm{GL}(\mathfrak{A})_{S}, \\
& \hat{r}(\gamma, t)=r_{t}(\gamma)=\gamma-t \cdot \pi_{S} \cdot \gamma \cdot \pi_{S^{\perp}} .
\end{aligned}
$$

(Again $r_{t}(\gamma)(S) \subset S, r_{t}(\gamma)\left(S^{\perp}\right) \subset S^{\perp}, r_{t}(\gamma) \cdot r_{t}\left(\gamma^{-1}\right)=1, r_{t}\left(\gamma^{-1}\right) \cdot r_{t}(\gamma)=1$, and thus $r_{t}$ is well defined.) Then $r_{0}=i d, r_{1}=i \cdot r$, where $i: \mathrm{GL}(\mathfrak{A})_{S, S^{\perp}} \rightarrow \mathrm{GL}(\mathfrak{A})_{S}$ is the inclusion. Further, $r_{t}(\gamma)=\gamma$ for $\gamma \in \mathrm{GL}(\mathfrak{U})_{S, S^{\perp}}$ and $0 \leqq t \leqq 1$. Thus, $\operatorname{GL}(\mathfrak{U})_{S, S^{\perp}} \subset \mathrm{GL}(\mathfrak{A})_{S}$ is a strong deformation retract of $\mathrm{GL}(\mathfrak{U})_{S}$.

Next we show that $\mathrm{U}(\mathfrak{Q})_{S} \subset \mathrm{GL}(\mathfrak{U})_{S, S^{\perp}}$ is a strong deformation retract of $\mathrm{GL}(\mathfrak{A})_{S, S^{\perp}}$. The map $u$ of Theorem 2.2 defines a deformation retract map

$$
u: \mathrm{GL}(\mathfrak{A})_{S, S^{\perp}} \rightarrow \mathrm{U}(\mathfrak{A})_{S},
$$

$u(\gamma)=\mu$, where $\gamma=\mu \cdot \kappa$ is the unique decomposition of Theorem 2.2.

$u$ is well defined. (By Corollary 2.2, $u(\gamma)(S)=S$, and hence $u(\gamma)\left(S^{\perp}\right)=S^{\perp}$. Therefore, $u(\gamma) \in \mathrm{U}(\mathfrak{U})_{S}$.) A deformation retract homotopy is given by

$$
\begin{aligned}
& \hat{u}: \mathrm{GL}(\mathfrak{A})_{S, S^{\perp}} \times[0,1] \rightarrow \mathrm{GL}(\mathfrak{U})_{S, S^{\perp}}, \\
& \hat{u}(\gamma, t)=u_{t}(\gamma)=\mu \cdot(t \cdot 1+(1-t) \cdot \kappa) .
\end{aligned}
$$

Again the map $u_{t}$ is well defined. (Namely $t \cdot 1+(1-t) \cdot \kappa \in \mathrm{GL}(\mathfrak{A})$ by Lemma 2.7,

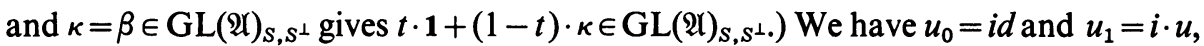
where $i: \mathrm{U}(\mathfrak{U})_{S} \rightarrow \mathrm{GL}(\mathfrak{A})_{S, S^{\perp}}$ is the inclusion. Finally, $u_{t}(\mu)=\mu$ for $\mu \in \mathrm{U}(\mathfrak{A})_{S}$ and $0 \leqq t \leqq 1$. Which proves the theorem.

Corollary 2.3. Let $\mathfrak{A}$ be a $C^{*}$-algebra with $1 \in \mathfrak{A}$. Then $\mathrm{U}(\mathfrak{A}) \subset \mathrm{GL}(\mathfrak{U})$ is a strong deformation retract of the space $\mathrm{GL}(\mathfrak{A})$. In particular, $\mathrm{U}(\mathfrak{A})$ and $\mathrm{GL}(\mathfrak{A})$ are of the same homotopy type. The strong deformation retract map

$$
\begin{aligned}
& u_{t}: \operatorname{GL}(\mathfrak{U}) \rightarrow \mathrm{GL}(\mathfrak{U}), \\
& u_{t}(\gamma)=\mu \cdot(t \cdot 1+(1-t) \cdot \kappa), \quad 0 \leqq t \leqq 1,
\end{aligned}
$$

of Theorem 2.3 has further the following property: If $S \subset H$ is a closed linear subspace, and if $\gamma_{1}, \gamma_{2} \in \mathrm{GL}(\mathfrak{H})$ with $\gamma_{i}\left(S^{\perp}\right)=\left(\gamma_{i}(S)\right)^{\perp}, i=1,2$, and with $\left.\gamma_{1}\right|_{s}=\left.\gamma_{2}\right|_{s}$, then $\left.u_{t}\left(\gamma_{1}\right)\right|_{s}=\left.u_{t}\left(\gamma_{2}\right)\right|_{s}$ for $0 \leqq t \leqq 1$.

Proof. We have only to observe that $\left.\kappa_{1}\right|_{S}=\left.\kappa_{2}\right|_{S}$ and $\left.\mu_{1}\right|_{S}=\left.\mu_{2}\right|_{s}$. Compare Corollary 2.2.

3. The metric space $\mathfrak{I}(H)$ of all closed linear subspaces of a Hilbert space $H$. Let $\mathfrak{I}(H)=\{S ; S \subset H$ and $S$ a closed linear subspace of $H\}$. We recall the definition of 
various metrics defined on $\mathfrak{T}(H)$ and some of their properties. If $x \in H$ and $S \in \mathfrak{I}(H)$, then $d(x, S)=\inf \{\|x-y\| ; y \in S\}=\left\|\left(1-\pi_{S}\right)(x)\right\|$.

Definition 3.1. For $S, T \in \mathfrak{T}(H)$ we define

$$
\begin{aligned}
& \delta(S, T)=\sup \{d(x, T) ; x \in S \text { and }\|x\|=1\}, \\
& d(S, T)=\delta(S, T)+\delta(T, S), \text { and } \\
& g(S, T)=\left\|\pi_{S}-\pi_{T}\right\| .
\end{aligned}
$$

LEMMA 3.1.

$0 \leqq \delta(S, T) \leqq 1$, and $0 \leqq g(S, T) \leqq 1$.

$\delta(S, T)=0$ if and only if $S \subset T$.

If $S \subset S^{\prime}$, and $T^{\prime} \subset T$, then $\delta(S, T) \leqq \delta\left(S^{\prime}, T^{\prime}\right)$.

$\delta(S, T)=\left\|\left(1-\pi_{T}\right) \cdot \pi_{S}\right\|$.

$\delta(S, T) \leqq \delta(S, R)+\delta(R, T)$.

$\delta(S, T)=\delta\left(T^{\perp}, S^{\perp}\right), g(S, T)=g\left(S^{\perp}, T^{\perp}\right)$.

$\delta(S, T) \leqq g(S, T)$ and $g(S, T) \leqq \delta(S, T)+\delta(T, S)$.

Proof. These properties follow immediately from the definitions. See, for example, [3].

COROLlaRY 3.1. The functions $d$ and $g$ on $\mathfrak{T}(H) \times \mathfrak{I}(H)$ define complete metrics on $\mathfrak{I}(H)$. We have

$$
g(S, T) \leqq d(S, T) \leqq 2 \cdot g(S, T) \quad \text { for } S, T \in \mathfrak{T}(H),
$$

and these two metrics are therefore equivalent.

Proof. The completeness is easily proved by using the $g$-metric.

Observe that $\delta$ itself does not define a metric.

The metric space $\mathfrak{T}(H)$ is the set $\mathfrak{T}(H)$ with the equivalence class of metrics containing $d$ and $g$.

LEMMA 3.2. Let $S, T \in \mathfrak{I}(H)$. Then the following relation holds

$$
(\delta(S, T))^{2}+\left(c\left(S, \pi_{T}\right)\right)^{2}=1 .
$$

In particular $\pi_{T}(S)$ is closed if and only if $\delta(S, T)<1$.

Proof. Observe that $\left\|\left(1-\pi_{T}\right)(x)\right\|^{2}=\|x\|^{2}-\left\|\pi_{T}(x)\right\|^{2}$ for $x \in H$. Therefore

$$
\begin{aligned}
(\delta(S, T))^{2} & =\sup \left\{\left\|\left(1-\pi_{T}\right)(x)\right\|^{2} ; x \in S \text { and }\|x\|=1\right\} \\
& =1-\inf \left\{\left\|\pi_{T}(x)\right\|^{2} ; x \in S \text { and }\|x\|=1\right\} \\
& =1-\left(c\left(S, \pi_{T}\right)\right)^{2} .
\end{aligned}
$$

Lemma 2.3 implies therefore $\pi_{T}(S)$ is closed if and only if $\delta(S, T)<1$. 
Of crucial importance is the following theorem established by T. Kato [8].

THEOREM 3.1. If $S, T \in \mathfrak{T}(H)$ with $\delta(S, T)<1$, then $T^{\prime}=\pi_{\mathbb{T}}(S)$ is in $\mathfrak{T}(H)$ and satisfies

$$
\delta\left(S, T^{\prime}\right)=\delta\left(T^{\prime}, S\right)=\delta(S, T)=g\left(S, T^{\prime}\right) .
$$

If further $\delta(T, S)<1$, then $T^{\prime}=T$.

Proof. From Lemma 3.2 follows $T^{\prime}=\pi_{T}(S)$ is closed and therefore in $\mathfrak{T}(H)$.

Since $\pi_{T} \cdot \pi_{S}=\pi_{T^{\prime}} \cdot \pi_{S}$, we have $\left(1-\pi_{T}\right) \cdot \pi_{S}=\left(1-\pi_{T^{\prime}}\right) \cdot \pi_{S}$ and therefore

$$
\delta(S, T)=\left\|\left(1-\pi_{T}\right) \cdot \pi_{S}\right\|=\left\|\left(1-\pi_{T^{\prime}}\right) \cdot \pi_{S}\right\|=\delta\left(S, T^{\prime}\right) .
$$

Consider the map $\alpha=\pi_{T^{\prime}} \cdot \pi_{S}$. Then $\alpha(H)=T^{\prime}$ is closed and we can apply Lemma 2.4 .

$$
c\left(\alpha(H), \alpha^{*}\right)=c\left(T^{\prime}, \pi_{S} \cdot \pi_{T^{\prime}}\right)=c\left(T^{\prime}, \pi_{S}\right)=c\left(\alpha^{*}(H), \pi_{T^{\prime}} \cdot \pi_{S}\right) .
$$

But $\alpha^{*}(H)=(\operatorname{kernel}(\alpha))^{\perp}=\left(S^{\perp}\right)^{\perp}=S$. Therefore

$$
c\left(T^{\prime}, \pi_{S}\right)=c\left(S, \pi_{T^{\prime}} \cdot \pi_{S}\right)=c\left(S, \pi_{T^{\prime}}\right)
$$

By Lemma 3.2 again $\delta\left(T^{\prime}, S\right)=\delta\left(S, T^{\prime}\right)$. We have $\delta\left(T^{\prime}, S\right)=\left\|\left(1-\pi_{S}\right) \cdot \pi_{T^{\prime}}\right\|$ $=\left\|\left(\pi_{T^{\prime}}-\pi_{S}\right) \cdot \pi_{T^{\prime}}\right\| \leqq\left\|\pi_{T^{\prime}}-\pi_{S}\right\|=g\left(S, T^{\prime}\right)$. On the other hand,

$$
\left(\pi_{S}-\pi_{T^{\prime}}\right)(x)=\left(1-\pi_{T^{\prime}}\right) \cdot \pi_{S}(x)-\pi_{T^{\prime}} \cdot\left(1-\pi_{S}\right)(x)
$$

for $x \in H$. We obtain

$$
\begin{aligned}
\left\|\left(\pi_{S}-\pi_{T^{\prime}}\right)(x)\right\|^{2} & =\left\|\left(1-\pi_{T^{\prime}}\right) \cdot \pi_{S}(x)\right\|^{2}+\left\|\pi_{T^{\prime}}\left(1-\pi_{S}\right)(x)\right\|^{2} \\
& \leqq \delta\left(S, T^{\prime}\right)^{2} \cdot\left\|\pi_{S}(x)\right\|^{2}+\delta\left(T^{\prime}, S\right)^{2} \cdot\left\|\left(1-\pi_{S}\right)(x)\right\|^{2}=\delta\left(S, T^{\prime}\right)^{2} \cdot\|x\|^{2} .
\end{aligned}
$$

And, therefore, $g\left(S, T^{\prime}\right)=\left\|\pi_{S}-\pi_{T^{\prime}}\right\| \leqq \delta\left(S, T^{\prime}\right)$. I.e., $g\left(S, T^{\prime}\right)=\delta\left(S, T^{\prime}\right)$. If further $\delta(T, S)<1$, then again $c\left(T, \pi_{S}\right)>0$, and therefore kernel $\left(\pi_{S} \cdot \pi_{T}\right)=T^{\perp}$. But also

$$
\operatorname{kernel}\left(\pi_{T} \cdot \pi_{S}\right)^{*}=\operatorname{kernel}\left(\pi_{S} \cdot \pi_{T}\right)=\left(\pi_{T} \cdot \pi_{S}(H)\right)^{\perp}=T^{\prime \perp} .
$$

Hence $T^{\perp}=T^{\perp}$, which implies $T^{\prime}=T$.

COROllary 3.2 .

$$
g(S, T)=\max \{\delta(S, T), \delta(T, S)\} \quad \text { for } S, T \in \mathfrak{T}(H) .
$$

If $g(S, T)<1$, then $\delta(S, T)=\delta(T, S)=g(S, T)$.

Proof. If $\delta(S, T)<1$ and $\delta(T, S)<1$, then by Theorem $3.1 g(S, T)=\delta(S, T)$ $=\delta(T, S)$. If $\delta(S, T)=1$ or $\delta(T, S)=1$, then $\delta(S, T), \delta(T, S) \leqq g(S, T) \leqq 1$ implies $g(S, T)=1$. For another proof see [1].

Corollary 3.3. Suppose $S, T \in \mathfrak{T}(H)$ with $\delta(S, T)<1$. Then there exists an $S^{\prime} \in \mathfrak{I}(H)$ with $S \subset S^{\prime}$ and $g\left(S^{\prime}, T\right)=\delta(S, T)$. 
Proof. We apply Theorem 3.1 to $\delta\left(T^{\perp}, S^{\perp}\right)=\delta(S, T)<1$. Let $\tilde{S}=\pi_{S^{\perp}}\left(T^{\perp}\right) \subset S^{\perp}$.

$$
g\left(\tilde{S}^{\perp}, T\right)=g\left(T^{\perp}, \tilde{S}\right)=\delta\left(T^{\perp}, S^{\perp}\right)=\delta(S, T) .
$$

Let $S^{\prime}=\tilde{S}^{\perp}$.

Lemma 3.3. Let $\alpha, \beta \in \mathrm{GL}(H)$ and let $S, T \in \mathfrak{T}(H)$. Then

$$
\begin{aligned}
& \delta(\alpha(S), \beta(T)) \leqq(\|\alpha-\beta\|+\delta(S, T)) \cdot\left\|\alpha^{-1}\right\|, \quad \text { and } \\
& d(\alpha(S), \beta(T)) \leqq(\|\alpha-\beta\|+d(S, T)) \cdot\left(\left\|\alpha^{-1}\right\|+\left\|\beta^{-1}\right\|\right) .
\end{aligned}
$$

Proof. By definition, $\delta(\alpha(S), \beta(T))=\sup \{d(\alpha(x), \beta(T)) ; x \in S$ and $\|\alpha(x)\|=1\}$, and $d(\alpha(x), \beta(T))=\inf \{\|\alpha(x)-\beta(y)\| ; y \in T\}$. We compute

$$
\begin{aligned}
\|\alpha(x)-\beta(y)\| & \leqq\|\alpha(x)-\beta(x)\|+\|\beta(x)-\beta(y)\| \\
& \leqq\|\alpha-\beta\| \cdot\|x\|+\|\beta\| \cdot\left\|\left[(\|x\|)^{-1} \cdot x-(\|x\|)^{-1} \cdot y\right]\right\| \cdot\|x\| .
\end{aligned}
$$

Therefore, $d(\alpha(x), \beta(T)) \leqq\|\alpha-\beta\| \cdot\|x\|+\|\beta\| \cdot \delta(S, T) \cdot\|x\|$. But $\|x\|=\left\|\alpha^{-1} \cdot \alpha(x)\right\|$ $\leqq\left\|\alpha^{-1}\right\| \cdot\|\alpha(x)\|=\left\|\alpha^{-1}\right\|$ for $\|\alpha(x)\|=1$. And we obtain

$$
\delta(\alpha(S), \beta(T)) \leqq(\|\alpha-\beta\|+\delta(S, T)) \cdot\left\|\alpha^{-1}\right\| .
$$

Lemma 3.4. Let $\alpha, \beta \in \mathfrak{L}(H), S \in \mathfrak{T}(H), c(S, \alpha)>0$, and let $\beta(S)$ be closed. Then

$$
\delta(\alpha(S), \beta(S)) \leqq c(S, \alpha)^{-1} \cdot\|\alpha-\beta\| .
$$

Proof. Again by definition

$$
\delta(\alpha(S), \beta(S))=\sup \{d(\alpha(x), \beta(S)) ; x \in S \text { and }\|\alpha(x)\|=1\},
$$

and $d(\alpha(x), \beta(S))=\inf \{\|\alpha(x)-\beta(y)\| ; y \in S\}$. In particular

$$
d(\alpha(x), \beta(S)) \leqq\|\alpha(x)-\beta(x)\| \leqq\|\alpha-\beta\| \cdot\|x\| .
$$

Since $1=\|\alpha(x)\| \geqq c(S, \alpha) \cdot\|x\|$, we obtain $\|x\| \leqq c(S, \alpha)^{-1}$. Therefore $\delta(\alpha(x), \beta(S))$ $\leqq c(S, \alpha)^{-1} \cdot\|\alpha-\beta\|$, which proves the lemma.

Lemma 3.5. Let $S, T \in \mathfrak{I}(H)$ with $g(S, T)<1$. Consider $\alpha_{t}=1-t \cdot \pi_{T^{\perp}} \in \mathfrak{Q}(H)$, $0 \leqq t \leqq 1$. Then $\alpha_{t}(S) \in \mathfrak{T}(H), 0 \leqq t \leqq 1$, and $t \rightarrow \alpha_{t}(S) \in \mathfrak{T}(H), 0 \leqq t \leqq 1$, is a continuous path in $\mathfrak{T}(H)$ which connects $S$ with $T$ such that

$$
g\left(S, \alpha_{t}(S)\right)=t \cdot g(S, T), \quad 0 \leqq t \leqq 1 .
$$

Proof. For $x \in H$ we compute

$$
\begin{aligned}
\left\|\alpha_{t}(x)\right\|^{2} & =\left(\left(1-t \cdot \pi_{T^{\perp}}\right)(x),\left(1-t \cdot \pi_{T^{\perp}}\right)(x)\right) \\
& =\left(\left(\pi_{T}+(1-t) \cdot \pi_{T^{\perp}}\right)(x),\left(\pi_{T}+(1-t) \cdot \pi_{T^{\perp}}\right)(x)\right) \\
& =\left\|\pi_{T}(x)\right\|^{2}+(1-t)^{2} \cdot\left\|\pi_{T^{\perp}}(x)\right\|^{2} \geqq\left\|\pi_{T}(x)\right\|^{2} .
\end{aligned}
$$


Therefore, $c\left(S, \alpha_{t}\right) \geqq c\left(S, \pi_{T}\right)=\left(1-\delta(S, T)^{2}\right)^{1 / 2}=\left(1-g(S, T)^{2}\right)^{1 / 2}>0$. By Lemma 2.3, then $\alpha_{t}(S) \in \mathfrak{T}(H)$ for $0 \leqq t \leqq 1$. Certainly $\alpha_{0}(S)=S$, and $\alpha_{1}(S)=T$ by Theorem 3.1.

The path $t \rightarrow \alpha_{t}(S) \in \mathfrak{T}(H)$ is continuous. Namely,

$$
\begin{aligned}
d\left(\alpha_{t_{1}}(S), \alpha_{t_{2}}(S)\right) & \leqq\left\|\alpha_{t_{1}}-\alpha_{t_{2}}\right\| \cdot\left(c\left(S, \alpha_{t_{1}}\right)^{-1}+c\left(S, \alpha_{t_{2}}\right)^{-1}\right) \\
& \leqq 2 \cdot\left(1-g(S, T)^{2}\right)^{-1 / 2} \cdot\left|t_{1}-t_{2}\right|
\end{aligned}
$$

(Lemma 3.4).

Finally, we prove $g\left(S, \alpha_{t}(S)\right)=t \cdot g(S, T), 0 \leqq t \leqq 1$, by a continuity argument. First we conclude from Lemma 3.4 and Corollary 3.2 that $\delta\left(S, \alpha_{t}(S)\right) \leqq\left\|\pi_{S}-\alpha_{t} \cdot \pi_{S}\right\|$ $=t \cdot \delta(S, T)=t \cdot g(S, T)$. For short, let $T_{t}=\alpha_{t}(S)$. We claim $\delta\left(T_{t}, S\right)=\delta\left(S, T_{t}\right)$ for $0 \leqq t \leqq 1$. [Namely, let $\sigma=\sup \left\{s ; 0 \leqq s \leqq 1\right.$ with $\delta\left(T_{t}, S\right)=\delta\left(S, T_{t}\right)$ for $\left.0 \leqq t \leqq s\right\}$. Suppose $0 \leqq \sigma<1$. Since $\delta$ is continuous on $\mathfrak{I}(H) \times \mathfrak{I}(H)$, also $\delta\left(T_{\sigma}, S\right)=\delta\left(S, T_{\sigma}\right)$ $=\sigma \cdot g(S, T)<1$, and there exists $\varepsilon>0$ with $\delta\left(T_{t}, S\right)<1$ and $\delta\left(S, T_{t}\right)<1$ for $0 \leqq t \leqq \sigma+\varepsilon$. By Corollary 3.2, $\delta\left(T_{t}, S\right)=\delta\left(S, T_{t}\right)$ for $0 \leqq t \leqq \sigma+\varepsilon$, which contradicts the definition of $\sigma$.] By Corollary 3.2, then $g\left(S, T_{t}\right)=\delta\left(T_{t}, S\right)=\delta\left(S, T_{t}\right)=t \cdot g(S, T)$ for $0 \leqq t \leqq 1$.

LEMMA 3.6. Let $S, T \in \mathfrak{I}(H)$ with $g(S, T)<1$. Then $\gamma=\pi_{T} \cdot \pi_{S}+\left(1-\pi_{T}\right) \cdot\left(1-\pi_{S}\right)$ $\in \mathrm{GL}(H)$ and $\gamma(S)=T$.

Proof. $g(S, T)<1$ implies $\delta(S, T)=\delta\left(T^{\perp}, S^{\perp}\right)<1$ and $\delta(T, S)=\delta\left(S^{\perp}, T^{\perp}\right)<1$. By Theorem 3.1, $\left.\gamma\right|_{S}$ is an isomorphism which maps $S$ onto $T$, and $\left.\gamma\right|_{S^{\perp}}$ is an isomorphism which maps $S^{\perp}$ onto $T^{\perp}$. This proves $\gamma \in \mathrm{GL}(H)$. Certainly

$$
\gamma(S)=\pi_{T}(S)=T \text {. }
$$

Lemma 3.7. Let $R, S, T \in \mathfrak{T}(H)$ with $R \subset S$ and $R \subset T$, and let $S^{\prime}=R^{\perp} \cap S$ and $T^{\prime}=R^{\perp} \cap T$. Then

$$
\delta\left(S^{\prime}, T^{\prime}\right)=\delta(S, T) \text { and } g\left(S^{\prime}, T^{\prime}\right)=g(S, T) .
$$

Proof. We have $\pi_{T}=\pi_{R}+\pi_{T^{\prime}}$ and $\pi_{S}=\pi_{R}+\pi_{S^{\prime}}$. Substitution into $\delta\left(S^{\prime}, T^{\prime}\right)$ $=\left\|\left(1-\pi_{T^{\prime}}\right) \cdot \pi_{S^{\prime}}\right\|$ and $g\left(S^{\prime}, T^{\prime}\right)=\left\|\pi_{S^{\prime}}-\pi_{T^{\prime}}\right\|$ proves immediately the lemma.

4. $R$-sets and maximal $R$-sets. We recall the well known Rellich criterion for compact linear maps of a Hilbert space which states that a linear map of a Hilbert space is compact if and only if for every $\varepsilon>0$ there is a closed linear subspace of finite codimension such that the norm of the restriction of the linear map to this subspace becomes smaller than $\varepsilon$. Various examples in analysis motivate a generalization of compact linear maps via the collection of closed linear subspaces of finite codimension in this criterion. A proper generalization of this collection is the concept of $R$-sets.

Definition 4.1. A subset $\Re \subset \mathfrak{T}(H)$ is called an $R$-set, if the following condition is satisfied: For any pair $S, T \in \Re$ and every $\varepsilon>0$ there exists an $R \in \Re$ with

$$
\delta(R, S)<\varepsilon \quad \text { and } \delta(R, T)<\varepsilon .
$$


EXAMPLES 4.1.

(1) Any subset $\Re \subset \mathfrak{T}(H)$ with the property that for any pair $S, T \in \Re$, also $S \cap T \in \Re$, is an $R$-set.

(2) The subset $\Re=\left\{S ; S \in \mathfrak{I}(H)\right.$ with $\left.S_{\min } \subset S\right\}$ of $\mathfrak{I}(H)$, where $S_{\min } \in \mathfrak{T}(H)$ is a fixed element, is an $R$-set.

(3) $\mathfrak{I}(H)$ itself is an $R$-set.

(4) Any set $\{S\}$ consisting of a single element $S \in \mathfrak{T}(H)$ only is an $R$-set.

(5) A set $\left\{S_{1}, \ldots, S_{n}\right\}$ consisting of $n$ elements of $\mathfrak{T}(H)$ is an $R$-set if and only if an $S_{i_{0}}=S_{\min } \subset S_{i}, i=1, \ldots, n$.

(6) $\mathfrak{T}(H)^{c}=\{S ; S \in \mathfrak{T}(H)$ and $\operatorname{codim}(S)<c\}$, where $c \geqq \boldsymbol{\aleph}_{0}$ is a cardinal number, is an $R$-set. (Namely, for $S, T \in \mathfrak{T}(H)^{c}$ also $S \cap T \in \mathfrak{T}(H)^{c}$. This follows from $S \cap T$ $=S \cap\left(\pi_{S}\left(T^{\perp}\right)\right)^{\perp}$ and hence $(S \cap T)^{\perp}=S^{\perp} \oplus \mathrm{Cl}\left(\pi_{S}\left(T^{\perp}\right)\right)$.) In particular:

$$
\mathfrak{I}(H)^{f}=\{S ; S \in \mathfrak{T}(H) \text { and codim }(S) \text { is finite }\} \text { is an } R \text {-set. }
$$

(7) If $\sigma=\left\{S_{n}\right\}_{n=1}^{\infty}$ is a sequence of closed linear subspaces with $S_{n+1} \subset S_{n}, n=1$, $2, \ldots$, then $\Re=\left\{S_{n} ; n=1,2, \ldots\right\}$ is an $R$-set.

(8) Further if $\sigma=\left\{S_{n}\right\}_{n=1}^{\infty}$ is a sequence of closed linear subspaces as in (7), then

$$
M(\sigma)=\left\{S ; S \in \mathfrak{T}(H) \text { with } \lim _{n \rightarrow \infty} \delta\left(S_{n}, S\right)=0\right\} \text { is an } R \text {-set. }
$$

(9) For explicit examples of $R$-sets in analysis we refer to [4].

Definition 4.2. For any subset $\mathfrak{S} \subset \mathfrak{T}(H)$ we define

$$
\begin{aligned}
\mathfrak{S}^{\vee} & =\{T ; T \in \mathfrak{T}(H) \text { such that there is an } R \in \Im \text { with } R \subset T\}, \\
\mathrm{Cl}(\mathfrak{S}) & =\text { the closure of } \subseteq \text { in the metric space } \mathfrak{T}(H) .
\end{aligned}
$$

LEMma 4.1. If $\Re$ is an $R$-set, then $\Re^{`}$ and $\mathrm{Cl}(\Re)$ are $R$-sets again.

Proof. If $S, T \in \Re^{`}$ and $\varepsilon>0$ are given, then there are $S^{\prime}, T^{\prime} \in \Re$ with $S^{\prime} \subset S$, $T^{\prime} \subset T$ and there is an $R \in \Re$ with $\delta\left(R, S^{\prime}\right)<\varepsilon, \delta\left(R, T^{\prime}\right)<\varepsilon$. But then also $\delta(R, S)$ $\leqq \delta\left(R, S^{\prime}\right)<\varepsilon, \delta(R, T) \leqq \delta\left(R, T^{\prime}\right)<\varepsilon$. If $S, T \in \mathrm{Cl}(\Re)$ and $\varepsilon>0$ are given, then there are $S^{\prime}, T^{\prime} \in \Re$ with $d\left(S^{\prime}, S\right)<\varepsilon / 2, d\left(T^{\prime}, T\right)<\varepsilon / 2$ and there is an $R \in \Re$ with $\delta\left(R, S^{\prime}\right)<\varepsilon / 2, \delta\left(R, T^{\prime}\right)<\varepsilon / 2$. We conclude $\delta(R, S) \leqq \delta\left(R, S^{\prime}\right)+\delta\left(S^{\prime}, S\right)<\varepsilon, \delta(R, T)$ $\leqq \delta\left(R, T^{\prime}\right)+\delta\left(T^{\prime}, T\right)<\varepsilon$.

LEMMA 4.2. For any subset $\Im \subset \mathfrak{T}(H)$ we have $\mathrm{Cl}(\Im)^{\vee} \subset \mathrm{Cl}\left(\Im^{\vee}\right)$.

Proof. Suppose $T \in \mathrm{Cl}(\Im)^{`}$. Then there is a $T^{\prime} \in \mathrm{Cl}(\Im)$ with $T^{\prime} \subset T$, and there is further a sequence $\left\{S_{n}\right\}_{n=1}^{\infty}, S_{n} \in \Im$, with $g\left(S_{n}, T^{\prime}\right)<1 / n$. But $\delta\left(S_{n}, T\right) \leqq \delta\left(S_{n}, T^{\prime}\right)$ $\leqq g\left(S_{n}, T^{\prime}\right)<1 / n$. By Corollary 3.3 there is an $S_{n}^{\prime}$ with $S_{n} \subset S_{n}^{\prime}$ and

$$
g\left(S_{n}^{\prime}, T\right)=\delta\left(S_{n}, T\right)<1 / n
$$

Since $S_{n}^{\prime} \in \Im^{\vee}$, this implies $T \in \mathrm{Cl}\left(\Im^{\vee}\right)$. 
Definition 4.3. A subset $\mathfrak{M} \subset \mathfrak{T}(H)$ is a maximal $R$-set, if it is an $R$-set and if

$$
\mathfrak{M}^{\vee}=\mathfrak{M} \quad \text { and } \quad \mathrm{Cl}(\mathfrak{M})=\mathfrak{M} \text {. }
$$

EXAMPLes 4.2. In Examples 4.1, the $R$-sets in (2), (3), (6), and (8) are maximal.

THEOREM 4.1. Each $R$-set $\Re$ is contained in a uniquely determined smallest maximal $R$-set $M(\Re)$.

Proof. Let $M(\Re)=\mathrm{Cl}\left(\Re^{`}\right)$. Then

$$
M(\Re)^{`}=\mathrm{Cl}\left(\Re^{\vee}\right)^{\vee} \subset \mathrm{Cl}\left(\Re^{\vee}\right)=\mathrm{Cl}\left(\Re^{\vee}\right)=M(\Re) \subset M(\Re)^{`}
$$

implies $M(\Re)^{\vee}=M(\Re)$. Of course $\mathrm{Cl}(M(\Re))=M(\Re)$. Obviously $M(\Re)$ is the smallest maximal $R$-set containing the $R$-set $\Re$.

In associating algebraic structures to an $R$-set there is a freedom to enlarge the $R$-set to a maximal $R$-set (see next chapter). This motivates the introduction of maximal $R$-sets. Hence it is sufficient to consider maximal $R$-sets only.

Repeatedly we will apply the following argument:

Lemma 4.3. Let $\mathfrak{M}$ be a maximal $R$-set, let $T \in \mathfrak{T}(H)$ and suppose for each $\varepsilon>0$ there exists an $S \in \mathfrak{M}$ with $\delta(S, T)<\varepsilon$, then $S \in \mathfrak{M}$.

Proof. Let $\varepsilon>0$ be given. Then, by Corollary 3.3, there exists for the $S$ of the hypothesis an $S^{\prime} \in \mathfrak{T}(H)$ with $S \subset S^{\prime}$ and $g\left(S^{\prime}, T\right)=\delta(S, T)<\varepsilon$. Since $S^{\prime} \in \mathfrak{M}$, this implies $T \in \overline{\mathfrak{M}}=\mathfrak{M}$.

LEMMA 4.4. The maximal $R$-set $\mathfrak{M}=\left\{S ; S \in \mathfrak{T}(H)\right.$ with $\left.S_{\min } \subset S\right\}$, where $S_{\min }$ $\in \mathfrak{T}(H)$ is a fixed element, has the following representation: We introduce the Hilbert space $H^{\prime}=\left(S_{\mathrm{min}}\right)^{\perp}$. Then the map

$$
\begin{aligned}
& c: \mathfrak{M} \rightarrow \mathfrak{T}\left(H^{\prime}\right), \\
& c(S)=\left(S_{\min }\right)^{\perp} \cap S,
\end{aligned}
$$

is an isometry onto $\mathfrak{I}\left(H^{\prime}\right)$ with respect to the g-metric and $\delta$-structure of $\mathfrak{M}$ and $\mathfrak{I}\left(H^{\prime}\right)$.

Proof. Certainly $c$ is bijective and Lemma 3.7.

THEOREM 4.2. Let $\mathfrak{M}$ be a maximal $R$-set. If there exists a finite-dimensional $S_{0} \in \mathfrak{M}$, then there is an $S_{\min } \in \mathfrak{M}$ such that

$$
\mathfrak{M}=\left\{S ; S \in \mathfrak{I}(H) \text { with } S_{\min } \subset S\right\} .
$$

Proof. Consider an $S_{\min } \in \mathfrak{M}$ such that $\operatorname{dim}\left(S_{\min }\right)$ is minimal. Let $S \in \mathfrak{M}$ be 
given. Since $\mathfrak{M}$ is an $R$-set there exists for each $n=1,2, \ldots$, an $R_{n} \in \mathfrak{M}$ such that

$$
\delta\left(R_{n}, S_{\mathrm{min}}\right)<\frac{1}{n}, \quad \delta\left(R_{n}, S\right)<\frac{1}{n} .
$$

By Corollary 3.3, there is an $R_{n}^{\prime} \in \mathfrak{M}$ with $R_{n} \subset R_{n}^{\prime}$ and

$$
g\left(R_{n}^{\prime}, S_{\mathrm{min}}\right)=\delta\left(R_{n}, S_{\mathrm{min}}\right)<\frac{1}{n} .
$$

By Theorem 3.1, $S_{\mathrm{min}}$ and $R_{n}^{\prime}$ are isomorphic. Since $\operatorname{dim}\left(S_{\mathrm{min}}\right) \leqq \operatorname{dim}\left(R_{n}\right)$ $\leqq \operatorname{dim}\left(R_{n}^{\prime}\right)=\operatorname{dim}\left(S_{\min }\right)$ and $\operatorname{dim}\left(S_{\min }\right)$ is finite, we have $R_{n}=R_{n}^{\prime}$.

We conclude

$$
\delta\left(S_{\min }, S\right) \leqq \delta\left(S_{\min }, R_{n}\right)+\delta\left(R_{n}, S\right)<\frac{2}{n}, \quad n=1,2, \ldots
$$

Therefore, $\delta\left(S_{\min }, S\right)=0$ and, by Lemma $3.1, S_{\min } \subset S$.

COROLlaRY 4.1. If $H$ is a finite-dimensional Hilbert space, then the maximal R-sets $\mathfrak{M}$ of $\mathfrak{T}(H)$ are the spaces $\mathfrak{T}\left(H^{\prime}\right), 0 \leqq \operatorname{dim}\left(H^{\prime}\right) \leqq \operatorname{dim}(H)$. The concept of maximal $R$-sets becomes in this case trivial, it does not introduce any new structure.

\section{5. $C^{*}$-algebras associated with $R$-sets.}

Definition 5.1. For an $R$-set $\Re$ we define (compare [4] and [5])

$$
\begin{aligned}
I(\Re)= & \{\alpha ; \alpha \in \mathfrak{L}(H) \text { such that for each } \varepsilon>0 \text { there is an } S \in \mathfrak{R} \\
& \text { with } \left.\left\|\alpha \cdot \pi_{S}\right\|<\varepsilon \text { and }\left\|\alpha^{*} \cdot \pi_{S}\right\|<\varepsilon\right\}, \text { and } \\
A(\mathfrak{R})= & \{\alpha ; \alpha \in \mathfrak{L}(H) \text { such that for each } S \in \mathfrak{R} \text { and } \varepsilon>0 \text { there is } \\
& \text { a } \left.T \in \mathfrak{R} \text { with }\left\|\left(1-\pi_{S}\right) \cdot \alpha \cdot \pi_{T}\right\|<\varepsilon \text { and }\left\|\left(1-\pi_{S}\right) \cdot \alpha^{*} \cdot \pi_{T}\right\|<\varepsilon\right\} .
\end{aligned}
$$

THEOREM 5.1. $I(\Re)$ is a $C^{*}$-algebra in $\mathfrak{L}(H), A(\Re)$ is a $C^{*}$-algebra with unit 1 in $\mathfrak{Q}(H), I(\Re) \subset A(\Re)$, and $I(\Re)$ is a closed twosided ${ }^{*}$-ideal in $A(\Re)$.

Proof. Immediately from the definitions, compare [4] and [5].

In [4] and [5] it was also shown that $I(\Re)=I(M(\Re))$ and $A(\Re)=A(M(\Re))$, where $M(\Re)$ is the uniquely determined maximal $R$-set associated with $\Re$. This implies that one can restrict oneself to maximal $R$-sets. The classical situation is obtained, if we choose as a $R$-set the maximal $R$-set $\mathfrak{M}=\mathfrak{T}(H)^{f}$ (Examples 4.1 (6)). Then

$$
\begin{aligned}
& I\left(\mathfrak{T}(H)^{f}\right)=\mathfrak{C}(H), \text { the ideal of compact linear maps in } \mathfrak{L}(H) . \\
& A\left(\mathfrak{T}(H)^{f}\right)=\mathfrak{L}(H) .
\end{aligned}
$$

Lemma 5.1. For $S \in \mathfrak{T}(H)$ let $\mathfrak{Q}(H)_{S^{\perp}}=\left\{\alpha ; \alpha \in \mathfrak{L}(H)\right.$ with $\alpha \cdot \pi_{S}=o$ and $\left.\pi_{S^{1}} \cdot \alpha=\alpha\right\}$. Certainly $\mathfrak{L}(H)_{S^{\perp}}$ is isometric to $\mathfrak{L}\left(S^{\perp}\right)$. If $\Re$ is an $R$-set and $S \in \Re$, then $\mathfrak{Q}(H)_{S^{\perp}} \subset I(\Re)$. In particular $\pi_{S^{\perp}} \in I(\Re)$, and hence $\pi_{S}, \pi_{S^{\perp}} \in A(\Re)$. 
Proof. A direct consequence of Definition 5.1.

Definition 5.2. Let $\Re$ be an $R$-set. Then we consider also the following closed invariant subgroups of the groups $\mathrm{GL}(A(\Re))$ and $\mathrm{U}(A(\Re))$ (Definition 2.2):

$$
\begin{aligned}
\mathrm{GL}_{q}(I(\Re)) & =\{1+\alpha ; 1+\alpha \in \mathrm{GL}(H) \text { and } \alpha \in I(\Re)\}, \quad \text { and } \\
\mathrm{U}_{q}(I(\Re)) & =\mathrm{GL}_{q}(I(\Re)) \cap \mathrm{U}(H) .
\end{aligned}
$$

Definition 5.3. If $S \in \mathfrak{T}(H)$ and $G \subset \mathrm{GL}(H)$ a subset, then we denote

$$
\begin{aligned}
G_{S} & =\{\gamma ; \gamma \in G \text { with } \gamma(S)=S\}, \\
G_{(S)} & =\left\{\gamma ; \gamma \in G \text { with }\left.\gamma\right|_{S}=i d\right\}, \quad \text { and } \\
\left.G\right|_{S} & =\left\{\left.\gamma\right|_{S} ; \gamma \in G \text { with } \gamma(S)=S\right\} .
\end{aligned}
$$

COROLlaRy 5.1. Let $\Re$ be an $R$-set, and $S \in \Re$. Then we have the representations

$$
\begin{aligned}
\mathrm{U}(A(\Re))_{S} & =\left.\mathrm{U}(A(\Re))\right|_{S} \times \mathrm{U}\left(S^{\perp}\right), \\
\mathrm{U}_{q}(I(\Re))_{S} & =\left.\mathrm{U}_{q}(I(\Re))\right|_{S} \times \mathrm{U}\left(S^{\perp}\right), \quad \text { and } \\
\mathrm{U}(A(\Re))_{\{S\}} & =\mathrm{U}_{q}(I(\Re))_{\{S\}}=\mathrm{U}\left(S^{\perp}\right) .
\end{aligned}
$$

Further $\mathrm{U}(A(\Re))_{S} \subset \mathrm{GL}(A(\Re))_{S}$, and $\mathrm{U}(I(\Re))_{S} \subset \mathrm{U}(I(\Re))_{S}$ are strong deformation retracts. In particular $\mathrm{U}(A(\Re))_{S}$ and $\mathrm{GL}(A(\Re))_{S}$, and $\mathrm{U}(I(\Re))_{S}$ and $\mathrm{GL}(I(\Re))_{S}$ are of the same homotopy type.

Proof. The first part follows directly from Lemma 5.1. The second part from Theorem 2.3 in the case of $A(\Re)$, and by the same construction as in the proof of Theorem 2.3 in the case of $I(\Re)$. (We have $\pi_{S^{\perp}} \in I(\Re)$ and $\pi_{S}=1-\pi_{S^{\perp}} \in A(\Re)$.)

Definition 5.4. Let $\subseteq \subset \mathfrak{T}(H)$. If $\gamma \in \mathrm{GL}(H)$, then let $\gamma(\mathfrak{S})=\{\gamma(S) ; S \in \mathfrak{S}\}$. We denote

$$
\begin{aligned}
\mathrm{GL}(\mathfrak{S}) & =\left\{\gamma ; \gamma \in \mathrm{GL}(H) \text { with } \gamma(\mathfrak{S})=\mathbb{S} \text { and } \gamma^{*}(\mathfrak{S})=\mathfrak{S}\right\}, \quad \text { and } \\
\mathrm{U}(\mathfrak{S}) & =\{\mu ; \mu \in \mathrm{U}(H) \text { with } \mu(\mathfrak{S})=\mathfrak{S}\} .
\end{aligned}
$$

If $\mathfrak{S}$ is closed in $\mathfrak{T}(H)$, then GL(S) and $\mathrm{U}(\mathfrak{S})$ are closed subgroups of $\mathrm{GL}(H)$.

LEMmA 5.2. Let $\Re$ be an $R$-set and let $\gamma \in \mathrm{GL}(H)$. Then $\gamma \in A(\Re)$ if and only if for each $S \in \Re$ we have $\gamma^{-1}(S) \in M(\Re)$ and $\gamma^{*-1}(S) \in M(\Re)$.

Proof. Observe first that by the definition of the adjoint map $\gamma(S)=\left(\gamma^{*-1}\left(S^{\perp}\right)\right)^{\perp}$ for any $S \in \mathfrak{I}(H)$. Suppose now $\gamma \in A(\Re)$. Let $S \in \Re$ and $\varepsilon>0$ be arbitrarily assigned. From Lemma 2.2 we have the representations

$$
\pi_{\gamma\left(S^{\perp}\right)}=\gamma \cdot \pi_{S^{\perp}} \cdot \beta \cdot \pi_{S^{\perp}} \cdot \gamma^{*} \text { and } \pi_{\gamma^{*}\left(S^{\perp}\right)}=\gamma^{*} \cdot \pi_{S^{\perp}} \cdot \tilde{\beta} \cdot \pi_{S^{\perp}} \cdot \gamma
$$


By the definition of $A(\Re)$ there is a $T \in \Re$ with

$$
\begin{aligned}
\left\|\left(1-\pi_{S}\right) \cdot \gamma \cdot \pi_{T}\right\| & <(\|\gamma\| \cdot\|\tilde{\beta}\|)^{-1} \cdot \varepsilon, \text { and } \\
\left\|\left(1-\pi_{S}\right) \cdot \gamma^{*} \cdot \pi_{T}\right\| & <(\|\gamma\| \cdot\|\beta\|)^{-1} \cdot \varepsilon .
\end{aligned}
$$

We conclude

$$
\delta\left(T, \gamma^{-1}(S)\right)=\delta\left(T,\left(\gamma^{*}\left(S^{\perp}\right)\right)^{\perp}\right)=\left\|\pi_{\gamma^{\star}\left(S^{\perp}\right)} \cdot \pi_{T}\right\|=\left\|\gamma^{*} \cdot \pi_{S^{\perp}} \cdot \widetilde{\beta} \cdot\left(\pi_{S^{\perp}} \cdot \gamma \cdot \pi_{T}\right)\right\|<\varepsilon,
$$

and similarly $\delta\left(T, \gamma^{*-1}(S)\right)=\delta\left(T,\left(\gamma^{\perp}\left(S^{\perp}\right)\right)^{\perp}\right)<\varepsilon$. By Lemma 4.3,

$$
\gamma^{-1}(S) \in M(\Re) \text { and } \gamma^{*-1}(S) \in M(\Re) \text {. }
$$

Suppose now $\gamma \in \mathrm{GL}(H)$ satisfies $\gamma^{-1}(S), \gamma^{*-1}(S) \in M(\Re)$ for all $S \in \Re$. We turn to the definition of $A(\Re)$ and assume $S \in \Re$ and $\varepsilon>0$ be given. Let $T^{\prime}=\gamma^{-1}(S)$ and $T^{\prime \prime}=\gamma^{*-1}(S)$. Since $\Re$ is an $R$-set, there is an $R \in \Re$ with $\delta\left(R, T^{\prime}\right)<(\|\gamma\|)^{-1} \cdot \varepsilon$ and $\delta\left(R, T^{\prime \prime}\right)<(\|\gamma\|)^{-1} \cdot \varepsilon$. Observe that with Lemma 2.2 we can conclude that $\pi_{S^{\perp}} \cdot \gamma \cdot \pi_{T^{\prime}}=0$ and $\pi_{S^{\perp}} \cdot \gamma^{*} \cdot \pi_{T^{m}}=0$. We compute

$$
\begin{aligned}
\left\|\left(1-\pi_{S}\right) \cdot \gamma \cdot \pi_{R}\right\| & \leqq\left\|\left(1-\pi_{S}\right) \cdot \gamma \cdot\left(1-\pi_{T^{\prime}}\right) \cdot \pi_{R}\right\|+\left\|\left(1-\pi_{S}\right) \cdot \gamma \cdot \pi_{T^{\prime}} \cdot \pi_{R}\right\| \\
& \leqq\|\gamma\| \cdot \delta\left(R, T^{\prime}\right)<\varepsilon,
\end{aligned}
$$

and similarly $\left\|\left(1-\pi_{S}\right) \cdot \gamma^{*} \cdot \pi_{R}\right\|<\varepsilon$. Which proves $\gamma \in A(\Re)$.

THEOREM 5.2 (COMPARE [5]). Let $\mathfrak{M}$ be a maximal $R$-set. Then

$$
\begin{aligned}
\mathrm{GL}(\mathfrak{M}) & =\mathrm{GL}(H) \cap A(\mathfrak{M})=\mathrm{GL}(A(\mathfrak{M})), \quad \text { and } \\
\mathrm{U}(\mathfrak{M}) & =\mathrm{U}(H) \cap A(\mathfrak{M})=\mathrm{U}(A(\mathfrak{M})) .
\end{aligned}
$$

Proof. If $\gamma \in \mathrm{GL}(\mathfrak{M})$, then $\gamma^{-1}(\mathfrak{M})=\mathfrak{M}$ and $\gamma^{*-1}(\mathfrak{M})=\mathfrak{M}$ and, by Lemma 5.2, $\gamma \in \mathrm{GL}(H) \cap A(\mathfrak{M})$. If $\gamma \in \mathrm{GL}(H) \cap A(\mathfrak{M})$, then by Corollary $2.1, \gamma^{-1} \in \mathrm{GL}(H)$ $\cap A(\mathfrak{M})$, and we conclude $\gamma(\mathfrak{M})=\mathfrak{M}$ and $\gamma^{*}(\mathfrak{M})=\mathfrak{M}$, and therefore $\gamma \in \mathrm{GL}(\mathfrak{M})$. This proves also $\mathrm{U}(\mathfrak{M})=\mathrm{U}(H) \cap A(\mathfrak{M})$.

LEMMA 5.3. Let $\mathfrak{M}$ be a maximal $R$-set. For any pair $S, T \in \mathfrak{M}$ and every $\varepsilon>0$ there exists an $R \in \mathfrak{M}$ with $R \subset S$ and $\delta(R, T)<\varepsilon$ (compare Definition 4.1).

Proof. We may assume $0<\varepsilon \leqq 1$. First there is an $\tilde{R} \in \mathfrak{M}$ with $\delta(\tilde{R}, S)<\varepsilon / 8$ and $\delta(\tilde{R}, T)<\varepsilon / 8$. By Corollary 3.3 there are $S^{\prime}, T^{\prime} \in \mathfrak{M}$ with $\tilde{R} \subset S^{\prime}, \tilde{R} \subset T^{\prime}$, and $g\left(S^{\prime}, S\right)<\varepsilon / 8, g\left(T^{\prime}, T\right)<\varepsilon / 8$. Consider now $\gamma=\pi_{S^{\prime}} \cdot \pi_{S^{\prime}}+\left(1-\pi_{S}\right) \cdot\left(1-\pi_{S^{\prime}}\right) \in \mathrm{GL}(\mathfrak{M})$ (Lemma 3.6). We have $\gamma\left(S^{\prime}\right)=S$, and we compute

$$
\|\gamma-1\|=\left\|\pi_{S^{\prime}} \cdot \pi_{S^{\prime}}-\pi_{S}-\pi_{S^{\prime}}+\pi_{S} \cdot \pi_{S^{\prime}}\right\| \leqq \delta\left(S, S^{\prime}\right)+\delta\left(S^{\prime}, S\right) \leqq 2 \cdot g\left(S^{\prime}, S\right)<\varepsilon / 4,
$$

and $\left\|\gamma^{-1}\right\|=\left\|(1+(\gamma-1))^{-1}\right\| \leqq 1 /(1-\|\gamma-1\|)<2$. Let $R=\gamma(\tilde{R}) \in \mathfrak{M}$. Certainly $R \subset \gamma\left(S^{\prime}\right)=S$. Now $\delta(R, T) \leqq \delta(\gamma(\tilde{R}), T)+\delta\left(T^{\prime}, T\right)$. But by Lemma 3.3, $\delta\left(\gamma(\tilde{R}), T^{\prime}\right)$ $\leqq\|\gamma-1\| \cdot\left\|\gamma^{-1}\right\|<\varepsilon / 2$. And $\delta\left(T^{\prime}, T\right) \leqq g\left(T^{\prime}, T\right)<\varepsilon / 8$. Hence $\delta(R, T)<\varepsilon$. 
Definition 5.5. Let $\mathfrak{S} \subset \mathfrak{I}(H)$ and let $S_{0} \in \mathfrak{T}(H)$. Then we denote

$$
\left.\mathfrak{S}\right|_{S_{0}}=\mathfrak{S} \cap \mathfrak{I}\left(S_{0}\right)=\left\{S ; S \in \mathfrak{S} \text { with } S \subset S_{0}\right\}
$$

THEOREM 5.3. Let $\mathfrak{M}$ be a maximal $R$-set and let $S_{0} \in \mathfrak{M}$. Then $\left.\mathfrak{M}\right|_{S_{0}}$ is a maximal $R$-set again with respect to the Hilbert space $S_{0}$.

Proof. Lemma 5.3 gives $\left.\mathfrak{M}\right|_{S_{0}}$ is an $R$-set. Evidently $\left(\left.\mathfrak{M}\right|_{S_{0}}\right)^{\vee}=\left.\mathfrak{M}\right|_{S_{0}}$ (see Definition 4.3). And $\left.\mathfrak{M}\right|_{S_{0}}=\mathfrak{M} \cap \mathfrak{T}\left(S_{0}\right)$ is closed, since $\mathfrak{T}\left(S_{0}\right) \subset \mathfrak{T}(H)$ is closed.

Definition 5.6. Let $\mathfrak{A} \subset \mathfrak{Q}(H)$ and $S_{0} \in \mathfrak{T}(H)$. Then we denote by

$$
\left.\mathfrak{A}\right|_{S_{0}}=\left\{\left.\alpha\right|_{S_{0}} ;\left.\alpha\right|_{S_{0}} \in \mathscr{L}\left(S_{0}\right) \text { where } \alpha \in \mathfrak{A} \text { with } \alpha\left(S_{0}\right) \subset S_{0}\right\} \text {. }
$$

THEOREM 5.4. Let $\mathfrak{M}$ be a maximal $R$-set and $S_{0} \in \mathfrak{M}$. Then

$$
I\left(\left.\mathfrak{M}\right|_{S_{0}}\right)=\left.I(\mathfrak{M})\right|_{S_{0}} \text { and } A\left(\left.\mathfrak{M}\right|_{S_{0}}\right)=\left.A(\mathfrak{M})\right|_{S_{0}} .
$$

Proof. Directly from Definition 5.1 with the use of Lemma 5.3.

THEOREM 5.5. Let $\mathfrak{M}$ be a maximal $R$-set and let $S \in \mathfrak{M}$. Then we have

$$
\begin{aligned}
\mathrm{GL}\left(A\left(\left.\mathfrak{M}\right|_{S}\right)\right) & =\mathrm{GL}(A(\mathfrak{M}))_{S} / \mathrm{GL}(A(\mathfrak{M}))_{\{S\}}, \\
\mathrm{U}\left(A\left(\left.\mathfrak{M}\right|_{S}\right)\right) & =\mathrm{U}(A(\mathfrak{M}))_{S} / \mathrm{U}(A(\mathfrak{M}))_{\{S\}}, \\
\mathrm{GL}_{q}\left(I\left(\left.\mathfrak{M}\right|_{S}\right)\right) & =\mathrm{GL}_{q}(I(\mathfrak{M}))_{S} / \mathrm{GL}_{q}(I(\mathfrak{M}))_{\{S\}}, \\
\mathrm{U}_{q}\left(I\left(\left.\mathfrak{M}\right|_{S}\right)\right) & =\mathrm{U}_{q}(I(\mathfrak{M}))_{S} / \mathrm{U}_{q}(I(\mathfrak{M}))_{\{S\}}, \\
\mathrm{U}(A(\mathfrak{M}))_{S} & =\mathrm{U}\left(A\left(\left.\mathfrak{M}\right|_{S}\right)\right) \times \mathrm{U}\left(S^{\perp}\right), \quad \text { and } \\
\mathrm{U}_{q}(I(\mathfrak{M}))_{S} & =\mathrm{U}_{q}\left(I\left(\left.\mathfrak{M}\right|_{S}\right)\right) \times \mathrm{U}\left(S^{\perp}\right) .
\end{aligned}
$$

Proof. Direct consequence of Theorem 5.4.

\section{The path components of maximal $R$-sets.}

LEMMA 6.1 (COMPARE [4]). Let $\mathfrak{M}$ be a maximal $R$-set. Each open ball $\mathfrak{B}_{(S, r)}$ $=\{T ; T \in \mathfrak{M}$ and $g(S, T)<r\}$ in $\mathfrak{M}$ with center $S$ and radius $r, 0<r \leqq 1$, is path connected.

Proof. Let $T \in \mathfrak{B}_{(S, r)}$ be given. We consider first $S^{\perp}, T^{\perp} \in \mathfrak{T}(H)$. Since $g\left(S^{\perp}, T^{\perp}\right)$ $=g(S, T)<r$, we can apply Lemma 3.5 to the pair $S^{\perp}, T^{\perp}$. Consider $\alpha_{t}=1-t \cdot \pi_{T}$ $\in \mathfrak{L}(H), 0 \leqq t \leqq 1$. Then $t \rightarrow \alpha_{t}\left(S^{\perp}\right) \in \mathfrak{T}(H)$ is a continuous path in $\mathfrak{T}(H)$, which connects $S^{\perp}$ with $T^{\perp}$ and with $g\left(S^{\perp}, \alpha_{t}\left(S^{\perp}\right)\right)=t \cdot g\left(S^{\perp}, T^{\perp}\right)$ for $0 \leqq t \leqq 1$.

Now we turn to the path $t \rightarrow T_{t}=\left(\alpha_{t}\left(S^{\perp}\right)\right)^{\perp} \in \mathfrak{I}(H), 0 \leqq t \leqq 1$. The complement map $\perp: \mathfrak{I}(H) \rightarrow \mathfrak{I}(H)$ is continuous by Lemma 3.1. The new path is therefore continuous again, and connects $S$ with $T$ in $\mathfrak{T}(H)$. We have $g\left(S, T_{t}\right)=g\left(S^{\perp}, \alpha_{t}\left(S^{\perp}\right)\right)$ $=t \cdot g(S, T)<r, 0 \leqq t \leqq 1$. 
Finally, $T_{t} \in \mathfrak{M}, 0 \leqq t \leqq 1$. We apply Lemma 4.3. Let $\varepsilon>0$ be given. By Lemma 2.2, $\pi_{\alpha_{t}\left(S^{\perp}\right)}=\alpha_{t} \cdot \pi_{S^{\perp}} \cdot \beta \cdot \pi_{S^{\perp}} \cdot \alpha_{t}^{*}$. There exists an $R \in \mathfrak{M}$ with $\delta(R, S)<\left(\left\|\alpha_{t}\right\| \cdot\|\beta\|\right)^{-1} \cdot \varepsilon$ and $\delta(R, T)<\left(\left\|\alpha_{t}\right\| \cdot\|\beta\|\right)^{-1} \cdot \varepsilon$. Then

$$
\begin{aligned}
\delta\left(R, T_{t}\right)=\delta\left(R,\left(\alpha_{t}\left(S^{\perp}\right)\right)^{\perp}\right) & =\left\|\left(1-\pi_{\left(\alpha_{t}\left(S^{\perp}\right)\right)^{\perp}}\right) \cdot \pi_{R}\right\|=\left\|\pi_{\alpha_{t}\left(S^{\perp}\right)} \cdot \pi_{R}\right\| \\
& \leqq\left\|\alpha_{t}\right\| \cdot\|\beta\| \cdot\left\|\pi_{S^{\perp}} \cdot\left((1-t) \cdot 1+t \cdot \pi_{T^{\perp}}\right) \cdot \pi_{R}\right\| \\
& \leqq\left\|\alpha_{t}\right\| \cdot\|\beta\| \cdot((1-t) \cdot \delta(R, S)+t \cdot \delta(R, T))<\varepsilon .
\end{aligned}
$$

Corollary 6.1. A maximal $R$-set $\mathfrak{M}$ is locally path connected. Path components and connected components coincide therefore, and they are open and closed in $\mathfrak{M}$.

Definition 6.1. First we introduce the following notation: If $G$ is a topological group, then $G^{\mathbf{1}}$ denotes the path component of its unit $1 \in G$. Let $\mathfrak{B}_{(s, 1)}$ $=\{T ; T \in \mathfrak{M}$ with $g(S, T)<1\}$ be the open ball in $\mathfrak{M}$ in the $g$-metric with radius 1 and center $S \in \mathfrak{M}$. Then we have the following cross-section map

$$
\begin{aligned}
& s: \mathfrak{B}_{(S, 1)} \rightarrow \mathrm{GL}_{q}^{1}(I(\mathfrak{M})) \subset \mathrm{GL}^{1}(\mathfrak{M}) \\
& s(T)=\pi_{T} \cdot \pi_{S}+\left(1-\pi_{T}\right) \cdot\left(1-\pi_{S}\right) .
\end{aligned}
$$

LEMma 6.2. The map $s$ is well defined, continuous, and satisfies $s(T)(S)=T$.

Proof. By Lemma 3.6, $s(T) \in \mathrm{GL}(H)$ and $s(T)(S)=\pi_{T}(S)=T$. Further, $s(T)$ $=\left(1-\pi_{T^{1}}\right) \cdot\left(1-\pi_{S^{\perp}}\right)+\pi_{T^{\perp}} \cdot \pi_{S^{\perp}} \in \mathrm{GL}_{q}(I(\mathfrak{M}))$. To show the continuity of $s$, we compute

$$
\begin{aligned}
\left\|s\left(T_{1}\right)-s\left(T_{2}\right)\right\| & =\left\|\pi_{T_{1}} \cdot \pi_{S}-\pi_{T_{2}} \cdot \pi_{S}+\pi_{T_{1}^{1}} \cdot \pi_{S^{\perp}}-\pi_{T_{2}} \cdot \pi_{S^{1}}\right\| \\
& \leqq\left\|\pi_{T_{1}}-\pi_{T_{2}}\right\|+\left\|\pi_{T_{1}^{1}}-\pi_{T_{2}^{1}}\right\|=2 \cdot g\left(T_{1}, T_{2}\right) .
\end{aligned}
$$

By Lemma $6.1, \mathfrak{B}_{(s, 1)}$ is path connected. Since $1=s(S) \in s\left(\mathfrak{B}_{(S, 1)}\right)$, it follows $s\left(\mathfrak{B}_{(s, 1)}\right) \subset \mathrm{GL}_{q}^{1}(I(\mathfrak{M}))$.

DEFINITION 6.2. We have the action of the group GL(M) on $\mathfrak{M}$

$$
\begin{aligned}
& a: \operatorname{GL}(\mathfrak{M}) \times \mathfrak{M} \rightarrow \mathfrak{M}, \\
& a(\gamma, S)=\gamma(S) .
\end{aligned}
$$

LEMMA 6.3. a is continuous. If $\mathfrak{E} \in \pi_{0}(\mathfrak{M})$ is a path component of $\mathfrak{M}$, we have in particular the action of $\mathrm{GL}^{1}(\mathfrak{M})$ on $\mathbb{E}$

$$
a: \mathrm{GL}^{1}(\mathfrak{M}) \times \mathfrak{C} \rightarrow \mathfrak{C} .
$$

And from this action the maps

$$
\begin{aligned}
& a_{y}: \mathbb{C} \rightarrow \mathfrak{C}, \\
& a_{y}(S)=\gamma(S) \text { for a fixed } \gamma \in \mathrm{GL}^{1}(\mathfrak{M}), \text { and } \\
& a_{S}: \mathrm{GL}^{1}(\mathfrak{M}) \rightarrow \mathfrak{C}, \\
& a_{S}(\gamma)=\gamma(S) \text { for a fixed } S \in \mathbb{C} .
\end{aligned}
$$


$a_{\gamma}$ is a homeomorphism of $\mathfrak{C}$ onto itself for each $\gamma \in \mathrm{GL}^{1}(\mathfrak{M}) . a_{\mathrm{S}}$ is a continuous map onto $\mathfrak{C}$. The same holds for the group $\mathrm{GL}_{q}^{1}(I(\mathfrak{M}))$.

Proof. Let $\left(\gamma_{1}, S_{1}\right),\left(\gamma_{2}, S_{2}\right) \in \operatorname{GL}(\mathfrak{M}) \times \mathfrak{M}$. Then from Lemma 3.3

$$
\begin{aligned}
d\left(a\left(\gamma_{1}, S_{1}\right), a\left(\gamma_{2}, S_{2}\right)\right) & =d\left(\gamma_{1}\left(S_{1}\right), \gamma_{2}\left(S_{2}\right)\right) \\
& \leqq\left(\left\|\gamma_{1}-\gamma_{2}\right\|+d\left(S_{1}, S_{2}\right)\right) \cdot\left(\left\|\gamma_{1}^{-1}\right\|+\left\|\gamma_{2}^{-1}\right\|\right),
\end{aligned}
$$

which proves the continuity of $a$, and therefore of $a_{y}$ and $a_{s} . a_{y}$ is a homeomorphism, since $a_{\gamma} \cdot a_{\gamma}^{-1}=i d$ and $a_{\gamma}^{-1} \cdot a_{\gamma}=i d$.

To show that $a_{S}$ is a map onto $\mathfrak{E}$, let $T \in \mathfrak{C}$ be given. Then there exists a continuous path $S_{t}, 0 \leqq t \leqq 1$, in $\mathbb{E}$, which connects $S_{0}=S$ with $S_{1}=T$. We can find an integer $N$ with $g\left(S_{k / N}, S_{(k+1) / N}\right)<1$ for $k=0,1, \ldots, N-1$. It is therefore enough to show that for $S, T \in \mathbb{C}$ with $g(S, T)<1$ there exists a $\gamma \in \mathrm{GL}^{1}(\mathfrak{M})$ with $\gamma(S)=T$. We can take $s(T) \in \mathrm{GL}^{1}(\mathfrak{M})$ of Lemma 6.1 as such an element $\gamma$.

LEMMA 6.4. The map $a_{S}: \mathrm{GL}^{1}(\mathfrak{M}) \rightarrow \mathfrak{C}$, where $S \in \mathfrak{C}$, is open. The same holds for $\mathrm{GL}_{q}^{1}(I(\mathfrak{M}))$.

Proof. If $\gamma \in \mathrm{GL}^{1}(\mathfrak{M})$, then we denote by $l_{\gamma}: \mathrm{GL}^{1}(\mathfrak{M}) \rightarrow \mathrm{GL}^{1}(\mathfrak{M})$ the left translation $l_{\gamma}(\alpha)=\gamma \cdot \alpha, \alpha \in \mathrm{GL}^{1}(\mathfrak{M})$. Observe that $a_{S}=a_{\gamma} \cdot a_{S} \cdot l_{\gamma^{-1}}$ for $\gamma \in \mathrm{GL}^{1}(\mathfrak{M})$. Also that $\mathfrak{B}_{(S, 1)} \subset \mathbb{C}$ and $\left(a_{s} \cdot s\right)(T)=T$ for all $T \in \mathfrak{B}_{(S, 1)}$ by Lemma 6.2.

Suppose now $\mathscr{D} \subset \mathrm{GL}^{1}(\mathfrak{M})$ is an open subset, and consider $\gamma \in \mathfrak{D}$. Then there is an open neighborhood $\mathfrak{D}_{1} \subset \mathrm{GL}^{1}(\mathfrak{M})$ of 1 with $\mathfrak{D}_{1} \subset l_{\gamma^{-1}}(\mathfrak{D}) \cap\left(a_{S}\right)^{-1}\left(\mathfrak{B}_{(S, 1)}\right)$. Now $a_{S}(\mathfrak{O})=\left(a_{y} \cdot a_{s} \cdot l_{\gamma}-1\right)(\mathfrak{D})$. But $s^{-1}\left(\mathfrak{D}_{1}\right) \subset a_{S}\left(\mathfrak{D}_{1}\right)$ (if $T \in s^{-1}\left(\mathfrak{D}_{1}\right)$, then $s(T) \in \mathfrak{D}_{1}$ and $T=\left(a_{S} \cdot s\right)(T)$; hence $\left.T \in a_{S}\left(\mathfrak{D}_{1}\right)\right)$. Further $S \in s^{-1}\left(\mathfrak{D}_{1}\right)$ (namely $\left.1 \in \mathfrak{D}_{1}\right)$. Therefore, $a_{S}(\gamma)=\gamma(S) \in a_{\gamma}\left(s^{-1}\left(\mathfrak{D}_{1}\right)\right) \subset a_{y}\left(a_{S}\left(l_{\gamma^{-1}}(\mathfrak{D})\right)\right)=a_{S}(\mathfrak{D})$. Since $a_{\gamma}$ is a homeomorphism, $a_{y}\left(s^{-1}\left(\mathfrak{D}_{1}\right)\right)$ is open, which proves the lemma.

Definition 6.3. A Banach manifold is a topological Hausdorff space such that each point has an open neighborhood homeomorphic to an open subset of a Banach space. (See for example [10] and [13].)

THEOREM 6.1. A maximal $R$-set $\mathfrak{M}$ is a Banach manifold. In particular the path components of $\mathfrak{M}$ are connected Banach manifolds.

Proof. Let $S \in \mathfrak{M}$ be given, and let $\mathfrak{L} \in \pi_{0}(\mathfrak{M})$ be the path component of $\mathfrak{M}$ with $S \in \mathfrak{L}$. We construct a coordinate system $(\mathfrak{u}, f)$ at $S$ : Consider the open map of the preceding Lemma 6.4

$$
\begin{aligned}
& a_{S}: G^{1}(\mathfrak{M}) \rightarrow \mathfrak{C}, \\
& a_{S}(\gamma)=\gamma(S) .
\end{aligned}
$$

Let $\tilde{\mathfrak{u}}=\left\{\gamma ; \gamma \in \mathrm{GL}^{1}(\mathfrak{M})\right.$ with $\left.\pi_{S}^{\prime} \cdot \gamma \cdot \iota_{S} \in \mathrm{GL}(S)\right\}$, where $\iota_{s}: S \rightarrow H$ is the inclusion and $\pi_{S}^{\prime}: H \rightarrow S$ is the projection. $\tilde{\mathfrak{u}}$ is open in $\mathrm{GL}^{1}(\mathfrak{M})$. (Proof: The map $p_{S}: \mathrm{GL}^{1}(\mathfrak{M}) \rightarrow$ 
$\mathfrak{L}(S)$ defined by $p_{S}(\alpha)=\pi_{S}^{\prime} \cdot \alpha \cdot \iota_{S}$ is continuous. $\mathrm{GL}(S)$ is open in $\mathfrak{L}(S)$, and $\tilde{\mathfrak{u}}$ $=\left(p_{S}\right)^{-1}(\mathrm{GL}(S))$.) We define $\mathfrak{U}=a_{S}(\tilde{\mathfrak{u}}) . \mathfrak{U}$ is open in $\mathfrak{S}$ and therefore in $\mathfrak{M}$.

Next let $A(\mathfrak{M})_{S, S^{\perp}}=\left\{\alpha ; \alpha \in A(\mathfrak{M})\right.$ with $\alpha \cdot \pi_{S}=\alpha$ and $\left.\pi_{S^{\perp}} \cdot \alpha=\alpha\right\} . A(\mathfrak{M})_{S, S^{\perp}}$ is a closed linear subspace of the Banach space $A(\mathfrak{M})$ and hence a Banach space.

Before we construct the coordinate homeomorphism $f$, we notice the following: Let

$$
\mathfrak{L}(H)_{S, S^{\perp}}=\left\{\alpha ; \alpha \in \mathfrak{L}(H) \text { with } \alpha \cdot \pi_{S}=\alpha \text { and } \pi_{S^{\perp}} \cdot \alpha=\alpha\right\} .
$$

If $\alpha \in \mathfrak{Q}(H)_{S, S^{1}}$, then $1+\alpha \in \mathrm{GL}(H)$ (namely $(1+\alpha) \cdot(1-\alpha)=1$ and $\left.(1-\alpha) \cdot(1+\alpha)=1\right)$. And if $\alpha_{1}, \alpha_{2} \in \mathfrak{Q}(H)_{S, S^{\perp}}$, then $\left(1+\alpha_{1}\right)(S)=\left(1+\alpha_{2}\right)(S)$ if and only if $\alpha_{1}=\alpha_{2}$.

Consider now the continuous map

$$
\begin{aligned}
\tilde{f}: \tilde{\mathfrak{u}} & \rightarrow A(\mathfrak{M})_{S, S^{\perp}}, \\
\tilde{f}(\gamma) & =\pi_{S^{\perp}} \cdot \gamma \cdot \pi_{S} \cdot\left(\pi_{S} \cdot \gamma \cdot \pi_{S}+\pi_{S^{\perp}}\right)^{-1} \cdot \pi_{S} \\
& =\gamma \cdot \pi_{S} \cdot\left(\pi_{S} \cdot \gamma \cdot \pi_{S}+\pi_{S^{\perp}}\right)^{-1} \cdot \pi_{S}-\pi_{S} .
\end{aligned}
$$

Observe that $(1+f(\gamma))(S)=\gamma(S)$. We define $f$ via the commutative diagram

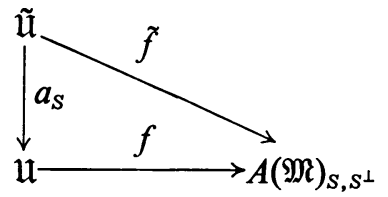

by $f=f \cdot\left(a_{S}\right)^{-1}$. The map $f$ is well defined and therefore continuous. (Namely if $a_{S}\left(\gamma_{1}\right)=a_{S}\left(\gamma_{2}\right)$ for $\gamma_{1}, \gamma_{2} \in \tilde{\mathfrak{u}}$, then $\gamma_{1}(S)=\gamma_{2}(S)$ and $\left(1+\tilde{f}\left(\gamma_{1}\right)\right)(S)=\left(1+\tilde{f}\left(\gamma_{2}\right)\right)(S)$, and hence $f\left(\gamma_{1}\right)=f^{f}\left(\gamma_{2}\right)$ by the remark above.)

Next we consider the continuous map

$$
\begin{aligned}
& \tilde{h}: A(\mathfrak{M})_{S, S^{\perp}} \rightarrow \mathrm{GL}^{1}(\mathfrak{M}), \\
& \tilde{h}(\alpha)=1+\alpha .
\end{aligned}
$$

$\tilde{h}$ is well defined. $(\tilde{h}(\alpha) \in \mathrm{GL}(H) \cap A(\mathfrak{M})=\mathrm{GL}(\mathfrak{M})$, and $1+t \cdot \alpha, 0 \leqq t \leqq 1$, is a path which connects 1 with $\tilde{h}(\alpha)$. Therefore, $\tilde{h}(\alpha) \in \mathrm{GL}^{\mathbf{1}}(\mathfrak{M})$ ).) Since $\pi_{S}^{\prime} \cdot(\tilde{h}(\alpha)) \cdot \iota_{S}=\mathbf{1}_{S}$, we have further $\tilde{h}(\alpha) \in \tilde{\mathfrak{u}}$. We compute

$$
\begin{aligned}
& (\tilde{f} \cdot \tilde{h})(\alpha)=\alpha \quad \text { for } \quad \alpha \in A(\mathfrak{M})_{S, s^{\perp}}, \quad \text { and } \\
& (\tilde{h} \cdot \tilde{f})(\gamma)=\mathbf{1}+\tilde{f}(\gamma) \quad \text { for } \quad \gamma \in \mathfrak{i} \mathrm{t} .
\end{aligned}
$$

And finally we define

$$
\begin{aligned}
& h: A(\mathfrak{M})_{S, S^{\perp}} \rightarrow \mathfrak{u}, \\
& h=a_{S} \cdot \tilde{h} .
\end{aligned}
$$

Then we have $(f \cdot h)(\alpha)=\alpha$ for $\alpha \in A(\mathfrak{M})_{S, S^{\perp}}$, and $(h \cdot f)(T)=(1+f(\gamma))(S)=\gamma(S)=T$ for $T \in \mathfrak{U}$, where $\gamma \in \tilde{\mathfrak{u}}$ with $a_{S}(\gamma)=\gamma(S)=T$. This proves $f: \mathfrak{U} \rightarrow A(\mathfrak{M})_{S, S^{\perp}}$ is a homeomorphism onto the Banach space $A(\mathfrak{M})_{S, S^{\perp}}$ and $h=f^{-1}$. 
Definition 6.4. Let $\mathfrak{M}$ be a maximal $R$-set, let $\mathfrak{E} \in \pi_{0}(\mathfrak{M})$ be a path component of $\mathfrak{M}$, and let $S \in \mathbb{C}$ be fixed. We consider the closed subgroup

$$
\mathrm{GL}^{1}(\mathfrak{M})_{S}=\left\{\gamma ; \gamma \in \mathrm{GL}^{1}(\mathfrak{M}) \text { with } \gamma(S)=S\right\}
$$

and we form the quotient space

$$
\mathrm{GL}^{1}(\mathfrak{M}) / \mathrm{GL}^{\mathbf{1}}(\mathfrak{M})_{S}=\left\{[\gamma] ; \gamma \in \mathrm{GL}^{1}(\mathfrak{M})\right\}
$$

of left cosets $[\gamma]=\gamma \cdot \mathrm{GL}^{\mathbf{1}}(\mathfrak{M})_{\mathrm{S}}$. This quotient space is given the topology induced by the projection map

$$
p: \mathrm{GL}^{1}(\mathfrak{M}) \rightarrow \mathrm{GL}^{1}(\mathfrak{M}) / \mathrm{GL}^{1}(\mathfrak{M})_{s} .
$$

We can lift the map $a_{S}: G^{1}(\mathfrak{M}) \rightarrow \mathfrak{E}$ onto the quotient space.

$$
\begin{aligned}
& \hat{a}_{S}: \mathrm{GL}^{1}(\mathfrak{M}) / \mathrm{GL}^{1}(\mathfrak{M})_{S} \rightarrow \mathfrak{C}, \\
& \hat{a}_{S}([\gamma])=a_{S}(\gamma)=\gamma(S) .
\end{aligned}
$$

Similarly for the group $\mathrm{GL}_{q}^{1}(I(\mathfrak{M}))$.

THEOREM 6.2. $\hat{a}_{S}: \mathrm{GL}^{1}(\mathfrak{M}) / \mathrm{GL}^{1}(\mathfrak{M})_{S} \rightarrow \mathfrak{C}$ is a homeomorphism. The spaces $\mathfrak{E}$ and $\mathrm{GL}^{1}(\mathfrak{M}) / \mathrm{GL}^{1}(\mathfrak{M})_{S}$ can therefore be identified.

Proof. Consider the commutative diagram

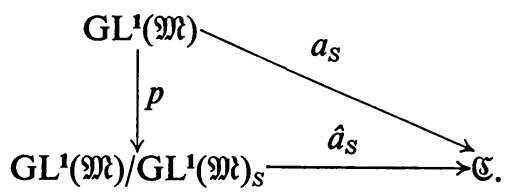

Certainly $\hat{a}_{S}$ is bijective. Since $a_{S}$ is continuous and open, $\hat{a}_{S}$ is a homeomorphism.

THEOREM 6.2 ${ }_{q} . \hat{a}_{S}: \mathrm{GL}_{q}^{1}(I(\mathfrak{M})) / \mathrm{GL}_{q}^{1}(I(\mathfrak{M}))_{S} \rightarrow \mathfrak{C}$ is a homeomorphism. The spaces (5 and $\mathrm{GL}_{q}^{\mathbf{1}}(\mathrm{I}(\mathfrak{M})) / \mathrm{GL}_{q}^{1}(I(\mathfrak{M}))_{S}$ can be identified also.

DEFINITION 6.5. We have a canonical local cross-section of $\mathrm{GL}^{1}(\mathfrak{M})_{S}$ in $\mathrm{GL}^{\mathbf{1}}(\mathfrak{M})$. $\mathfrak{B}^{\prime}=\left(\hat{a}_{S}\right)^{-1}\left(\mathfrak{B}_{(S, 1)}\right)$ is an open neighborhood of the point $[1] \in \mathrm{GL}^{1}(\mathfrak{M}) / \mathrm{GL}^{1}(\mathfrak{M})_{S}$. We define

$$
\begin{aligned}
& s^{\prime}: \mathfrak{B}^{\prime} \rightarrow \mathrm{GL}^{1}(\mathfrak{M}) \\
& s^{\prime}=s \cdot \hat{a}_{S}, \quad \text { i.e., } \quad s^{\prime}([\gamma])=s(\gamma(S)) .
\end{aligned}
$$

We have $p \cdot s^{\prime}([\gamma])=[s(\gamma(S))]$. But $(s(\gamma(S)))(S)=\gamma(S)$ and therefore $[s(\gamma(S))]=[\gamma]$. Or $p \cdot s^{\prime}([\gamma])=[\gamma]$ for $[\gamma] \in \mathfrak{B}^{\prime}$. Similarly for $\mathrm{GL}_{q}^{1}(I(\mathfrak{M}))_{S}$ and $\mathrm{GL}^{1}(I(\mathfrak{M}))$.

The Steenrod construction [14, p. 30] can now be applied, and we obtain:

THEOREM 6.3. If $K$ is a closed subgroup of $\mathrm{GL}^{1}(\mathfrak{M})_{S}$,

$$
p: \mathrm{GL}^{\mathbf{1}}(\mathfrak{M}) / K \rightarrow \mathfrak{C}=\mathrm{GL}^{1}(\mathfrak{M}) / \mathrm{GL}^{\mathbf{1}}(\mathfrak{M})_{S}
$$


the projection map induced by the inclusion of cosets, then a bundle structure can be assigned to $\mathrm{GL}^{1}(\mathfrak{M}) / K$ relative to $p$ such that the fibre of the bundle is $\mathrm{GL}^{1}(\mathfrak{M})_{S} / K$ and the group of the bundle is $\mathrm{GL}^{1}(\mathfrak{M})_{S} / K_{0}$ acting on $\mathrm{GL}^{1}(\mathfrak{M})_{S} / K$ as left translations, where $K_{0}$ is the largest subgroup of $K$ invariant in $\mathrm{GL}^{1}(\mathfrak{M})_{S}$. The same holds for the group $\mathrm{GL}^{1}(I(\mathfrak{M}))$.

COROLLARY 6.2. GL'(M) is a principal fibre bundle over

$$
\mathfrak{C}=\mathrm{GL}^{\mathbf{1}}(\mathfrak{M}) / \mathrm{GL}^{\mathbf{1}}(\mathfrak{M})_{S}
$$

with fibre and structure group $\mathrm{GL}^{\mathbf{1}}(\mathfrak{M})_{S}$, which acts on the fibres by left translations. In particular we have an exact sequence for the homotopy groups

$$
\cdots \rightarrow \pi_{n+1}(\mathfrak{E}) \rightarrow \pi_{n}\left(\mathrm{GL}^{1}(\mathfrak{M})_{S}\right) \rightarrow \pi_{n}\left(\mathrm{GL}^{1}(\mathfrak{M})\right) \rightarrow \pi_{n}(\mathfrak{E}) \rightarrow \cdots
$$

COROLlaRY $6.2_{q}$. GL $\mathrm{GL}_{q}^{1}(I(\mathfrak{M}))$ is a principal fibre bundle over

$$
\mathfrak{C}=\mathrm{GL}_{q}^{1}(I(\mathfrak{M})) / \mathrm{GL}_{q}^{1}(I(\mathfrak{M}))_{S}
$$

with fibre and structure group $\mathrm{GL}_{q}^{1}(I(\mathfrak{M}))_{S}$, which acts on the fibres by left translations. In particular we have an exact sequence for the homotopy groups

$$
\cdots \rightarrow \pi_{n+1}(\mathfrak{S}) \rightarrow \pi_{n}\left(\mathrm{GL}_{q}^{1}(I(\mathfrak{M}))_{S}\right) \rightarrow \pi_{n}\left(\mathrm{GL}_{q}^{1}(I(\mathfrak{M})) \rightarrow \pi_{n}(\mathfrak{E}) \rightarrow \cdots\right.
$$

The preceding constructions and arguments can also be applied to the unitary groups $U^{1}(\mathfrak{M})$ and $U_{q}^{1}(I(\mathfrak{M}))$.

Definition 6.1'. Let $\mathfrak{B}_{(S, 1)}=\{T ; T \in \mathfrak{M}$ with $g(S, T)<1\}$ be again the open ball in $\mathfrak{M}$ in the $g$-metric with radius 1 and center $S \in \mathfrak{M}$. Then we have the crosssection map

$$
\begin{aligned}
& v: \mathfrak{B}_{(S, 1)} \rightarrow \mathrm{U}_{q}^{1}(I(\mathfrak{M})) \subset \mathrm{U}^{1}(\mathfrak{M}), \\
& v=u \cdot s, \quad \text { i.e., } \quad v(T)=u\left(\pi_{T} \cdot \pi_{S}+\left(1-\pi_{T}\right) \cdot\left(1-\pi_{S}\right)\right)
\end{aligned}
$$

where $u: G L(\mathfrak{M}) \rightarrow \mathrm{U}(\mathfrak{M})$ is the map of Theorem 2.2 .

LEMMA 6.2'. The map $v$ is well defined, continuous, and satisfies $v(T)(S)=T$.

Proof. $v(T)(S)=T$ follows from Corollary 2.2. We show

$$
u\left(\mathrm{GL}_{q}^{1}(I(\mathfrak{M})) \subset \mathrm{GL}_{q}^{1}(I(\mathfrak{M})) .\right.
$$

Recall that if $\gamma \in \mathrm{GL}_{q}^{1}(I(\mathfrak{M}))$, then $u(\gamma)=\mu=\gamma \cdot \beta^{-1}$, where $\beta \in \mathrm{GL}(\mathfrak{M})$ is the uniquely determined positive and self-adjoint element with $\beta^{2}=\gamma^{*} \cdot \gamma$. We write $\beta=1+\alpha, \alpha \in A(\mathfrak{M})$, and show $\alpha \in I(\mathfrak{M})$. First $\beta^{2}=1+2 \alpha+\alpha^{2} \in \mathrm{GL}_{q}^{1}(I(\mathfrak{M}))$ implies $2 \alpha+\alpha^{2}=2 \alpha \cdot\left(1+\frac{1}{2} \alpha\right) \in I(\mathfrak{M})$. We have now $1+\frac{1}{2} \alpha \in \mathrm{GL}(\mathfrak{M})$. (Namely $1+\beta \in \mathrm{GL}(\mathfrak{M})$ by Lemma 2.7 , and therefore $1+\frac{1}{2} \alpha=\frac{1}{2} \cdot(1+\beta) \in G L(\mathfrak{M})$.) Since $I(\mathfrak{M})$ is an ideal in $A(\mathfrak{M})$, we conclude that $\alpha \in I(\mathfrak{M})$. This shows that $\beta$ and hence $\mu=\gamma \cdot \beta^{-1}$ are in $\mathrm{GL}_{q}^{\mathbf{1}}(I(\mathfrak{M}))$. 
From here on, Definition 6.2, Lemma 6.3, Lemma 6.4, Definition 6.4, and Definition 6.5 carry over word by word to the unitary groups $U^{1}(\mathfrak{M})$ and $U_{q}^{1}(I(\mathfrak{M}))$, and we obtain:

THEOREM 6.2'. $\hat{a}_{S}: \mathrm{U}^{1}(\mathfrak{M}) / \mathrm{U}^{1}(\mathfrak{M})_{S} \rightarrow \mathbb{C}$ is a homeomorphism. The spaces $\mathbb{C}$ and $\mathrm{U}^{1}(\mathfrak{M}) / \mathrm{U}^{1}(\mathfrak{M})_{S}$ can therefore be identified.

THEOREM $6.2_{q}^{\prime}$. $\hat{a}_{S}: \mathrm{U}_{q}^{1}(I(\mathfrak{M})) / \mathrm{U}_{q}^{1}(I(\mathfrak{M}))_{S} \rightarrow \mathbb{C}$ is a homeomorphism. The spaces and $\mathrm{U}_{q}^{1}(I(\mathfrak{M})) / \mathrm{U}_{q}^{1}(I(\mathfrak{M}))_{S}$ can be identified also.

THEOREM 6.3'. If $K$ is a closed subgroup of $\mathrm{U}^{\mathbf{1}}(\mathfrak{M}){ }_{S}$,

$$
p: \mathrm{U}^{\mathbf{1}}(\mathfrak{M}) / K \rightarrow \mathfrak{C}=\mathrm{U}^{\mathbf{1}}(\mathfrak{M}) / \mathrm{U}^{\mathbf{1}}(\mathfrak{M})_{S}
$$

the projection map induced by the inclusion of cosets, then a bundle structure can be assigned to $\mathrm{U}^{1}(\mathfrak{M}) / K$ relative to $p$ such that the fibre of the bundle is $\mathrm{U}^{1}(\mathfrak{M})_{S} / K$ and the group of the bundle is $\mathrm{U}^{1}(\mathfrak{M})_{S} / K_{0}$ acting on $\mathrm{U}^{1}(\mathfrak{M})_{S} / K$ as left translations, where $K_{0}$ is the largest subgroup of $K$ invariant in $\mathrm{U}^{1}(\mathfrak{M})_{s}$. The same holds for the group $\mathrm{U}_{q}^{1}(I(\mathfrak{M}))$.

COROLlaRY $6.2^{\prime}$. $\mathrm{U}^{1}(\mathfrak{M})$ is a principal fibre bundle over

$$
\mathfrak{C}=\mathrm{U}^{1}(\mathfrak{M}) / \mathrm{U}^{\mathbf{1}}(\mathfrak{M R})_{S}
$$

with fibre and structure group $\mathrm{U}^{1}(\mathfrak{M})_{S}$, which acts on the fibres by left translations. In particular we have the exact sequence for the homotopy groups

$$
\cdots \rightarrow \pi_{n+1}(\mathfrak{S}) \rightarrow \pi_{n}\left(\mathrm{U}^{1}(\mathfrak{M})_{S}\right) \rightarrow \pi_{n}\left(\mathrm{U}^{1}(\mathfrak{M})\right) \rightarrow \pi_{n}(\mathfrak{E}) \rightarrow \cdots
$$

COROLlaRY $6.2_{q}^{\prime}$. $\mathrm{U}_{q}^{1}(I(\mathfrak{M}))$ is a principal fibre bundle over

$$
\mathfrak{C}=\mathrm{U}_{q}^{1}(I(\mathfrak{M})) / \mathrm{U}_{q}^{1}(I(\mathfrak{M}))_{S}
$$

with fibre and structure group $\mathrm{U}_{q}^{1}(I(\mathfrak{M}))_{S}$, which acts on the fibres by left translations. In particular, we have the exact sequence for the homotopy groups

$$
\cdots \rightarrow \pi_{n+1}(\mathfrak{夭}) \rightarrow \pi_{n}\left(\mathrm { U } _ { q } ^ { 1 } ( I ( \mathfrak { M } ) _ { S } ) \rightarrow \pi _ { n } \left(\mathrm{U}_{q}^{1}(I(\mathfrak{M})) \rightarrow \pi_{n}(\mathfrak{E}) \rightarrow \cdots\right.\right.
$$

7. Stiefel spaces associated with maximal $R$-sets. In the following we introduce homogeneous spaces, which are direct analogues of the Stiefel manifolds of finitedimensional vectorspaces. We consider the group $\mathrm{GL}(A(\mathfrak{M}))$ only. Everything holds also for the group $\mathrm{GL}_{q}(I(\mathfrak{M}))$.

Definition 7.1. Let $\mathfrak{M}$ be a maximal $R$-set, $\mathfrak{E} \in \pi_{0}(\mathfrak{M})$ a path component of $\mathfrak{M}$, and let $S \in \mathbb{C}$ be fixed. The Stiefel space $\mathrm{St}(\mathbb{E})_{S}$ associated with the path component ( (relative to $S$ ) is defined as

$$
\operatorname{St}(\mathfrak{E})_{S}=\left\{\lambda ; \lambda \in \mathfrak{L}(S, H) \text { such that there is a } \gamma \in \mathrm{GL}^{1}(\mathfrak{M}) \text { with } \lambda=\gamma \cdot \iota_{S}\right\},
$$


where $\iota_{S}: S \rightarrow H$ is the inclusion. $\operatorname{St}(\mathbb{E})_{S}$ is a closed subset of $\mathfrak{L}(S, H)$ and is given the relative topology.

LEMMA 7.1. If $S_{1}, S_{2} \in \mathfrak{C}$, then the spaces $\mathrm{St}(\mathfrak{E})_{S_{1}}$ and $\mathrm{St}(\mathfrak{E})_{S_{2}}$ are homeomorphic. The reference element $S \in \mathbb{C}$ is therefore irrelevant. The Stiefel spaces $\operatorname{St}(\mathbb{E})_{S}$ are path connected.

Proof. There exists a $\beta \in G^{1}(\mathfrak{M})$ with $\beta\left(S_{1}\right)=S_{2}$ (Lemma 6.3). Consider the $\operatorname{map} i_{\beta}: \operatorname{St}(\mathfrak{E})_{S_{1}} \rightarrow \operatorname{St}(\mathfrak{E})_{S_{2}}, i_{\beta}(\lambda)=\gamma \cdot \beta^{-1} \cdot \iota_{S_{2}}$, where $\lambda=\gamma \cdot \iota_{S_{1}}$. Then $i_{\beta}$ is a homeomorphism. $\mathrm{GL}^{1}(\mathfrak{M})$ is path connected implies directly $\mathrm{St}(\mathbb{E})_{S}$ is path connected.

Definition 7.2. We have the natural projection map

$$
\begin{aligned}
& q: \operatorname{St}(\mathbb{C})_{S} \rightarrow \mathbb{C}, \\
& q(\lambda)=\lambda(S) .
\end{aligned}
$$

$q$ is continuous (Lemma 3.4) and maps $\mathrm{St}(\mathbb{E})_{S}$ onto $\mathbb{E}$.

Definition 7.3. Let $\mathfrak{O}\left(\iota_{S}\right)=q^{-1}\left(\mathfrak{B}_{(S, 1)}\right)=\left\{\lambda ; \lambda \in \operatorname{St}\left(\mathbb{E}_{S}\right.\right.$ with $\left.g(S, \lambda(S))<1\right\}$. We have the cross-section map

$$
\begin{aligned}
& r: \mathfrak{O}\left(\iota_{S}\right) \rightarrow \mathrm{GL}^{1}(\mathfrak{M}) \\
& r(\lambda)=\gamma \cdot \pi_{S}+\left(1-\pi_{\gamma(S)}\right) \cdot\left(1-\pi_{S}\right), \quad \text { where } \lambda=\gamma \cdot \iota_{S} .
\end{aligned}
$$

LEMma 7.2. $r$ is well defined, continuous, and satisfies $r(\lambda) \cdot \iota_{S}=\lambda$.

Proof. The continuity follows from Lemma 3.4.

DefinItion 7.4. We have the action of the group $\mathrm{GL}^{1}(\mathfrak{M})$ on $\mathrm{St}(\mathbb{E})_{S}$

$$
\begin{aligned}
& b: \operatorname{GL}^{1}(\mathfrak{M}) \times \operatorname{St}(\mathfrak{E})_{S} \rightarrow \operatorname{St}(\mathfrak{E})_{S}, \\
& b(\gamma, \lambda)=\gamma \cdot \lambda .
\end{aligned}
$$

$b$ is continuous. It determines the maps

$$
\begin{aligned}
& b_{\gamma}: \operatorname{St}(\mathfrak{C})_{S} \rightarrow \operatorname{St}(\mathfrak{C})_{S}, \\
& b_{\gamma}(\lambda)=\gamma \cdot \lambda \text { for a fixed } \gamma \in \mathrm{GL}^{1}(\mathfrak{M}), \text { and } \\
& b_{\lambda}: \mathrm{GL}^{1}(\mathfrak{M}) \rightarrow \operatorname{St}(\mathfrak{C})_{S}, \\
& b_{\lambda}(\gamma)=\gamma \cdot \lambda \text { for a fixed } \lambda \in \operatorname{St}(\mathfrak{C})_{S} .
\end{aligned}
$$

$b_{\gamma}$ is a homeomorphism of $\operatorname{St}(\mathfrak{E})_{S}$ onto itself for each $\gamma \in \mathrm{GL}^{1}(\mathfrak{M}) . b_{\lambda}$ is a continuous map onto $\mathrm{St}(\mathbb{E})_{S}$.

LEMMA 7.3. The map $b_{\lambda}: \mathrm{GL}^{1}(\mathfrak{M}) \rightarrow \mathrm{St}\left(\mathfrak{C}_{S}\right.$ is open.

Proof. First $b_{\iota_{s}}$ is open by the same arguments as in Lemma 6.4 using the 
cross-section $r$ of Definition 7.3. And $b_{\lambda}=b_{\iota_{\mathrm{S}}} \cdot k_{\beta}$, where $\lambda=\beta \cdot \iota_{\mathrm{S}}$ and $k: \mathrm{GL}^{1}(\mathfrak{M )}) \rightarrow$ $\mathrm{GL}^{1}(\mathfrak{M})$, the right translation $k_{\beta}(\gamma)=\gamma \cdot \beta$.

THEOREM 7.1. The Stiefel spaces $\mathrm{St}(\mathfrak{(})_{S}$ are Banach manifolds.

Proof. There is a natural connection with Theorem 6.1. In the following we refer to the notation introduced in the proof of this theorem. We construct a coordinate system $(\mathfrak{B}, g)$ at the point $\iota_{s}$. Consider the open map of the preceding Lemma 7.3

$$
\begin{aligned}
& b_{\iota_{s}}: \mathrm{GL}^{1}(\mathfrak{M}) \rightarrow \operatorname{St}(\mathbb{E})_{S}, \\
& b_{\iota_{s}}(\gamma)=\gamma \cdot \iota_{s} .
\end{aligned}
$$

Let $\tilde{\mathfrak{u}}=\left\{\gamma ; \gamma \in \mathrm{GL}^{1}(\mathfrak{M})\right.$ with $\left.\left.\left(\pi_{S} \cdot \gamma\right)\right|_{S}=\pi_{S}^{\prime} \cdot \gamma \cdot \iota_{S} \in \mathrm{GL}^{1}\left(\left.\mathfrak{M}\right|_{S}\right)\right\}$. $\tilde{\mathfrak{u}}$ is an open subset of $\mathrm{GL}^{1}(\mathfrak{M})$. Let $\mathfrak{B}=b_{\iota_{S}}(\tilde{\mathfrak{u}})$. Then $\mathfrak{B}$ is open in $\operatorname{St}(\mathfrak{S})_{S}$.

Consider next the continuous map

$$
\begin{aligned}
& \tilde{g}: \tilde{\mathfrak{u}} \rightarrow A(\mathfrak{M})_{S, S^{\perp}} \times \mathrm{GL}^{1}\left(\left.\mathfrak{M}\right|_{S}\right) \\
& \tilde{g}(\gamma)=\left(\tilde{f}(\gamma), \pi_{S}^{\prime} \cdot \gamma \cdot \iota_{S}\right) .
\end{aligned}
$$

We define $g$ via the commutative diagram

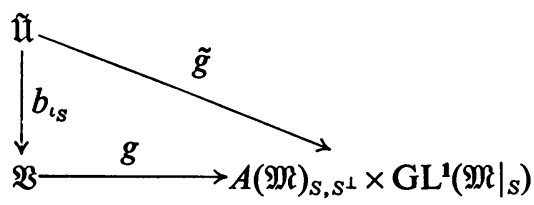

by $g=\tilde{g} \cdot\left(b_{t_{s}}\right)^{-1}$. The map $g$ is well defined and therefore continuous. (Namely if $b_{\iota_{s}}\left(\gamma_{1}\right)=b_{\iota_{s}}\left(\gamma_{2}\right)$ for $\gamma_{1}, \gamma_{2} \in \tilde{\mathfrak{u}}$, then $\gamma_{1} \cdot \iota_{S}=\gamma_{2} \cdot \iota_{S}$ and $\tilde{f}\left(\gamma_{1}\right)=\tilde{f}\left(\gamma_{2}\right)$, and therefore $\tilde{\boldsymbol{g}}\left(\gamma_{1}\right)=\tilde{g}\left(\gamma_{2}\right)$.)

We consider the continuous map

$$
\begin{aligned}
& \tilde{k}: A(\mathfrak{M})_{S, S^{\perp}} \times G^{1}\left(\left.\mathfrak{M}\right|_{S}\right) \rightarrow \mathrm{GL}^{1}(\mathfrak{M}), \\
& \tilde{k}\left(\alpha, \gamma^{\prime}\right)=(1+\alpha) \cdot\left(\iota_{S} \cdot \gamma^{\prime} \cdot \pi_{S}^{\prime}+\pi_{S^{1}}\right) .
\end{aligned}
$$

$\tilde{k}$ is well defined and continuous. Further $\pi_{S}^{\prime} \cdot \tilde{k}\left(\alpha, \gamma^{\prime}\right) \cdot \iota_{S}=\gamma^{\prime}$ implies $\tilde{k}\left(\alpha, \gamma^{\prime}\right) \in \mathfrak{u}$. We compute

$$
\begin{aligned}
& (\tilde{g} \cdot \tilde{k})\left(\alpha, \gamma^{\prime}\right)=\left(\alpha, \gamma^{\prime}\right) \quad \text { for } \quad\left(\alpha, \gamma^{\prime}\right) \in A(\mathfrak{M})_{S, S^{\perp}} \times G^{1}\left(\left.\mathfrak{M}\right|_{S}\right) \text {, and } \\
& (\tilde{k} \cdot \tilde{g})(\gamma)=\gamma \cdot \pi_{S}+\pi_{S^{\perp}} \quad \text { for } \quad \gamma \in \tilde{\mathfrak{u}} .
\end{aligned}
$$

Finally we define

$$
\begin{aligned}
& k: A(\mathfrak{M})_{S, S^{\perp}} \times \operatorname{GL}^{1}\left(\left.\mathfrak{M}\right|_{S}\right) \rightarrow \mathfrak{B}, \\
& k=b_{\iota_{s}} \cdot \tilde{k}
\end{aligned}
$$


Then we have $(g \cdot k)\left(\alpha, \gamma^{\prime}\right)=\left(\alpha, \gamma^{\prime}\right)$ for $\left(\alpha, \gamma^{\prime}\right) \in A(\mathfrak{M})_{S, S^{\perp}} \times \mathrm{GL}^{1}\left(\left.\mathfrak{M}\right|_{S}\right)$, and $(k \cdot g)(\lambda)$ $=\left(\gamma \cdot \pi_{S}+\pi_{S^{\perp}}\right) \cdot \iota_{S}=\lambda$ for $\lambda \in \mathfrak{B}$, where $\gamma \in \mathfrak{\mathfrak { u }}$ with $\gamma \cdot \iota_{S}=\lambda$. This proves $g: \mathfrak{B} \rightarrow$ $A(\mathfrak{M})_{S, S^{\perp}} \times \mathrm{GL}^{1}\left(\left.\mathfrak{M}\right|_{S}\right)$ is a homeomorphism onto the open subset $A(\mathfrak{M})_{S, S^{\perp}}$ $\times \mathrm{GL}^{1}\left(\left.\mathfrak{M}\right|_{S}\right)$ of the Banach space $A(\mathfrak{M})_{S, S^{\perp}} \times A\left(\left.\mathfrak{M}\right|_{S}\right)$.

Definition 7.5. Let $\mathfrak{M}$ be a maximal $R$-set, let $\mathfrak{C} \in \pi_{0}(\mathfrak{M})$ be a path component of $\mathfrak{M}$, and let $S \in \mathbb{C}$ be fixed. We consider the closed subgroup

$$
\mathrm{GL}^{1}(\mathfrak{M})_{\{S\}}=\left\{\gamma ; \gamma \in \mathrm{GL}^{1}(\mathfrak{M}) \text { and }\left.\gamma\right|_{s}=i d\right\},
$$

and we form the quotient space

$$
\mathrm{GL}^{\mathbf{1}}(\mathfrak{M}) / \mathrm{GL}^{\mathbf{1}}(\mathfrak{M})_{(S)}=\left\{[\gamma] ; \gamma \in \mathrm{GL}^{\mathbf{1}}(\mathfrak{M})\right\}
$$

of left cosets $[\gamma]=\gamma \cdot \mathrm{GL}^{1}(\mathfrak{M})_{\{S\}}$. This quotient space is given the topology induced by the projection map

$$
p: \mathrm{GL}^{1}(\mathfrak{M}) \rightarrow \mathrm{GL}^{1}(\mathfrak{M}) / \mathrm{GL}^{1}(\mathfrak{M})_{(S)} .
$$

We can lift the map $b_{\iota_{S}}: \mathrm{GL}^{1}(\mathfrak{M}) \rightarrow \operatorname{St}\left(\mathfrak{S}_{S}\right.$ onto the quotient space.

$$
\begin{aligned}
& \hat{b}_{\iota_{s}}: \operatorname{GL}^{1}(\mathfrak{M}) / G^{1}(\mathfrak{M})_{(S)} \rightarrow \operatorname{St}(\mathfrak{E})_{S}, \\
& \hat{b}_{\iota_{s}}([\gamma])=b_{\iota_{s}}(\gamma)=\gamma \cdot \iota_{S} .
\end{aligned}
$$

THEOREM 7.2. $\hat{b}_{t_{s}}: \mathrm{GL}^{1}(\mathfrak{M}) / \mathrm{GL}^{1}(\mathfrak{M})_{\{S\}} \rightarrow \operatorname{St}(\mathbb{E})_{S}$ is a homeomorphism. The spaces $\operatorname{St}(\mathbb{E})_{S}$ and $\mathrm{GL}^{1}(\mathfrak{M}) / \mathrm{GL}^{1}(\mathfrak{M})_{\{S\}}$ can be identified.

Proof. The same as that of Theorem 6.2.

Definition 7.6. We have a canonical local cross-section of $\mathrm{GL}^{1}(\mathfrak{M})_{(S)}$ in $\mathrm{GL}^{1}(\mathfrak{M})$. $\mathfrak{D}^{\prime}=\left(\hat{b}_{\iota_{s}}\right)^{-1}\left(\mathfrak{D}\left(\iota_{S}\right)\right)$ is an open neighborhood of the point $\left.[1] \in \mathrm{GL}^{1}(\mathfrak{M}) / \mathrm{GL}^{1}(\mathfrak{M})\right)_{(S)}$. We define

$$
\begin{aligned}
& r^{\prime}: \mathfrak{D}^{\prime} \rightarrow \mathrm{GL}^{1}(\mathfrak{M}), \\
& r^{\prime}=r \cdot \hat{b}_{\iota_{s}}, \quad \text { i.e., } \quad r^{\prime}([\gamma])=r\left(\gamma \cdot \iota_{s}\right) .
\end{aligned}
$$

We have $p \cdot r^{\prime}([\gamma])=\left[r\left(\gamma \cdot \iota_{S}\right)\right]$. But $r\left(\gamma \cdot \iota_{S}\right) \cdot \iota_{S}=\gamma \cdot \iota_{S}$ and therefore $\left[r\left(\gamma \cdot \iota_{S}\right)\right]=[\gamma]$. Hence $p \cdot r^{\prime}([\gamma])=[\gamma]$ for $[\gamma] \in \mathfrak{D}^{\prime}$.

The Steenrod construction $[14$, p. 30$]$ can be applied, and we obtain:

THEOREM 7.3. If $K$ is a closed subgroup of $\mathrm{GL}^{1}(\mathfrak{M})_{(S)}$,

$$
p: \mathrm{GL}^{1}(\mathfrak{M}) / K \rightarrow \mathrm{St}(\mathfrak{E})_{S}=\mathrm{GL}^{1}(\mathfrak{M}) / \mathrm{GL}^{1}(\mathfrak{M})_{\{S\}}
$$

the projection map induced by the inclusion of cosets, then a bundle structure can be assigned to $\mathrm{GL}^{1}(\mathfrak{M})_{(S)} / K$ and the group of the bundle is $\mathrm{GL}^{1}(\mathfrak{M})_{(S)} / K_{0}$ acting on $\mathrm{GL}^{1}(\mathfrak{M})_{(S)} / K$ as left translations, where $K_{0}$ is the largest subgroup of $K$ invariant in $\mathrm{GL}^{\mathbf{1}}(\mathfrak{M})_{\{S\}}$. 
COROLLARY 7.1. GL'(M) is a principal fibre bundle over

$$
\operatorname{St}(\mathfrak{E})_{S}=G^{1}(\mathfrak{M}) / G^{1}(\mathfrak{M})_{(S)}
$$

with fibre and structure group $\mathrm{GL}^{1}(\mathfrak{M})_{\{S\}}$ which acts on the fibres by left translations.

COROLlaRY 7.2. The projection

$$
q: \mathrm{St}(\mathbb{C})_{S}=\mathrm{GL}^{1}(\mathfrak{M}) / \mathrm{GL}^{1}(\mathfrak{M})_{\{S\}} \rightarrow \mathbb{C}=\mathrm{GL}^{1}(\mathfrak{M}) / \mathrm{GL}^{1}(\mathfrak{M})_{S}
$$

(compare Definition 7.2) defines a principal bundle over $\mathfrak{E}$ with fibre and structure group $\mathrm{GL}^{1}\left(\left.\mathfrak{M}\right|_{S}\right)=\mathrm{GL}^{1}(\mathfrak{M})_{S} / \mathrm{GL}^{1}(\mathfrak{M})_{(S)}$, which acts on the fibres by left translations.

The same constructions and arguments apply also to the orthogonal case.

Definition 7.1'. Let $\mathfrak{M}$ be a maximal $R$-set, let $\mathfrak{E} \in \pi_{0}(\mathfrak{M})$ be a path component of $\mathfrak{M}$, and let $S \in \mathbb{C}$ be fixed. The orthogonal Stiefel space $\mathrm{U}(\mathfrak{E})_{S}$ associated with the path component $\mathbb{C}$ (relative to $S$ ) is defined as

$$
\mathrm{U}(\mathbb{E})_{S}=\left\{\nu ; \nu \in \mathfrak{R}(S, H) \text { such that there is a } \mu \in \mathrm{U}^{1}(\mathfrak{M}) \text { with } \nu=\mu \cdot \iota_{S}\right\},
$$

where $\iota_{S}: S \rightarrow H$ is the inclusion. $\mathrm{U}(\mathfrak{C})_{S}$ is a closed subset of $\mathfrak{L}(S, H)$ and is given the relative topology.

LEMma 7.1'. If $S_{1}, S_{2} \in \mathbb{E}$, then the spaces $\mathrm{U}(\mathfrak{E})_{S_{1}}$ and $\mathrm{U}(\mathbb{E})_{S_{2}}$ are homeomorphic. The reference element $S \in \mathbb{C}$ is therefore irrelevant. The orthogonal Stiefel spaces $\mathrm{U}\left(\mathcal{C}_{S}\right)_{S}$ are path connected.

Proof. The same as of Lemma 7.1.

Definition 7.2'. We have the natural projection map

$$
\begin{aligned}
& q: \mathrm{U}(\mathfrak{C})_{S} \rightarrow \mathfrak{C}, \\
& q(\nu)=\nu(S) .
\end{aligned}
$$

$q$ is continuous and maps $\mathrm{U}(\mathbb{\complement})_{S}$ onto $\mathbb{E}$.

Definition 7.3'. Let $\mathfrak{B}\left(\iota_{S}\right)=q^{-1}\left(\mathfrak{B}_{(S, 1)}\right)=\left\{\nu ; \nu \in \mathrm{U}(\mathfrak{C})_{S}\right.$ with $\left.g(S, \nu(S))<1\right\}$. We have the cross-section map

$$
\begin{aligned}
& w: \mathfrak{B}\left(\iota_{S}\right) \rightarrow \mathrm{U}^{1}(\mathfrak{M}), \\
& w=u \cdot r, \quad \text { i.e., } \quad w(\nu)=u\left(\mu \cdot \pi_{S}+\left(1-\pi_{\mu(S)}\right) \cdot\left(1-\pi_{S}\right)\right) \quad \text { where } \nu=\mu \cdot \iota_{S},
\end{aligned}
$$

where $u: G L(\mathfrak{M}) \rightarrow \mathrm{U}(\mathfrak{M})$ is from Theorem 2.2 , and $r$ is the cross-section map of Definition 7.3.

LEMMA 7.2'. $w$ is well defined, continuous, and satisfies $w(v) \cdot \iota_{S}=\nu$.

Proof. The last property follows from Corollary 2.2.

Definition 7.4 and Lemma 7.3 carry over word by word to the orthogonal case. 
THEOREM 7.1'. The orthogonal Stiefel spaces $\mathrm{U}(\mathfrak{\complement})_{\mathrm{S}}$ are Banach manifolds.

Proof. The same construction as in Theorem 7.1. The group $\mathrm{GL}^{\mathbf{1}}(\mathfrak{M})$ is replaced by the group $\mathrm{U}^{\mathbf{1}}(\mathfrak{M})$, which is a Banach manifold. (The well-known exponential map exp: $H(A(\mathfrak{M})) \rightarrow \mathrm{U}^{1}(\mathfrak{M})$, where $H(A(\mathfrak{M}))$ is the closed real linear subspace of the hermitian elements of $A(\mathfrak{M})$, determines a homeomorphism from an open neighborhood of $o$ in $H(A(\mathfrak{M}))$ to an open neighborhood of 1 in $\mathrm{U}^{1}(\mathfrak{M})$. The inverse to the exponential map is provided by a log-map.)

Definitions 7.5 and 7.6 carry over again without any change to the orthogonal case. We conclude:

THEOREM 7.2'. $\hat{b}_{\iota_{S}}: \mathrm{U}^{1}(\mathfrak{M}) / \mathrm{U}^{1}(\mathfrak{M})_{(S)} \rightarrow \mathrm{U}(\mathbb{E})_{S}$ is a homeomorphism. The spaces $\mathrm{U}(\mathbb{E})_{S}$ and $\mathrm{U}^{1}(\mathfrak{M}) / \mathrm{U}^{1}(\mathfrak{M})_{\{S\}}$ can be identified.

THEOREM 7.3'. If $K$ is a closed subgroup of $\mathrm{U}^{1}(\mathfrak{M})_{\{S\}}$,

$$
p: \mathrm{U}^{1}(\mathfrak{M}) / K \rightarrow \mathrm{U}(\mathfrak{E})_{S}=\mathrm{U}^{1}(\mathfrak{M}) / \mathrm{U}^{1}(\mathfrak{M})_{(S)}
$$

the projection map induced by the inclusion of cosets, then a bundle structure can be assigned to $\mathrm{U}^{1}(\mathfrak{M}) / K$ relative to $p$ such that the fibre of the bundle is $\mathrm{U}^{1}(\mathfrak{M})_{(S)} / K$ and the group of the bundle is $\mathrm{U}^{1}(\mathfrak{M})_{(S)} / K_{0}$ acting on $\mathrm{U}^{1}(\mathfrak{M})_{(S)} / K$ as left translations, where $K_{0}$ is the largest subgroup of $K$ invariant in $\mathrm{U}^{1}(\mathfrak{M})_{\{S\}}$.

COROLlaRY 7.1'. U' $\mathrm{U}^{1}(\mathfrak{M})$ is a principal fibre bundle over $\mathrm{U}(\mathfrak{E})_{S}=\mathrm{U}^{1}(\mathfrak{M}) / \mathrm{U}^{1}(\mathfrak{M})_{\{S\}}$ with fibre and structure group $\mathrm{U}^{1}(\mathfrak{M})_{\{S\}} \subset \mathrm{U}\left(S^{\perp}\right)$, which acts on the fibres by left translations.

COROLlaRY 7.2'. The projection map

$$
q: \mathrm{U}(\mathfrak{C})_{S}=\mathrm{U}^{1}(\mathfrak{M}) / \mathrm{U}^{1}(\mathfrak{M})_{\{S\}} \rightarrow \mathbb{C}=\mathrm{U}^{1}(\mathfrak{M}) / \mathrm{U}^{1}(\mathfrak{M})_{S}
$$

defines a principal bundle over $\mathfrak{E}$ with fibre and structure group

$$
\mathrm{U}^{1}\left(\left.\mathfrak{M}\right|_{S}\right)=\mathrm{U}^{1}(\mathfrak{M})_{S} / \mathrm{U}^{1}(\mathfrak{M})_{\{S\}},
$$

which acts on the fibres by left translations.

THEOREM 7.4. The orthogonal Stiefel space $\mathrm{U}(\mathbb{E})_{S} \subset \mathrm{St}(\mathbb{E})_{S}$ is a strong deformation retract of the Stiefel space $\mathrm{St}(\mathbb{E})_{S}$. In particular $\mathrm{U}(\mathfrak{E})_{S}$ and $\mathrm{St}(\mathbb{E})_{S}$ are of the same homotopy type.

Proof. We introduce first the map

$$
\begin{aligned}
& e: \mathrm{GL}(\mathfrak{M}) \rightarrow \mathrm{GL}(\mathfrak{M}), \\
& e(\gamma)=\gamma \cdot \pi_{S}+\pi_{(\gamma(S))^{\perp}} \cdot \gamma \cdot \pi_{S^{\perp}}=\gamma-\pi_{\gamma(S)} \cdot \gamma \cdot \pi_{S^{\perp}} .
\end{aligned}
$$


$e$ is well defined. Namely we compute

$$
\begin{aligned}
& \left(\gamma-\pi_{\gamma(S)} \cdot \gamma \cdot \pi_{S^{\perp}}\right) \cdot\left(\gamma^{-1}+\pi_{S^{\prime}} \cdot \gamma^{-1} \cdot \pi_{(\gamma(S))^{\perp}}\right)=1, \quad \text { and } \\
& \left(\gamma^{-1}+\pi_{S^{\prime}} \cdot \gamma^{-1} \cdot \pi_{(\gamma(S))^{\perp}}\right) \cdot\left(\gamma-\pi_{\gamma(S)} \cdot \gamma \cdot \pi_{S^{\perp}}\right)=1 .
\end{aligned}
$$

This proves $e(\gamma) \in \mathrm{GL}(\mathfrak{M})$. The continuity of $e$ follows from Lemma 2.3.

Finally we consider the map

$$
\begin{aligned}
& j: \mathrm{GL}^{\mathbf{1}}(\mathfrak{M}) \times[0,1] \rightarrow \mathrm{GL}^{1}(\mathfrak{M}), \\
& j(\gamma, t)=j_{t}(\gamma)=u_{t}(e(\gamma)), \quad 0 \leqq t \leqq 1
\end{aligned}
$$

where $u_{t}$ is the strong deformation retract map of Corollary 2.3. We turn to the commutative diagram

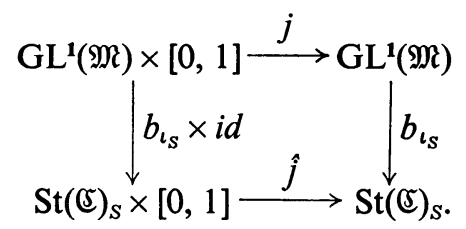

Let $\hat{j}=b_{\iota s} \cdot j \cdot\left(b_{\iota s} \times i d\right)^{-1}$. The map $\hat{j}$ is well defined by the particular property of $u_{t}$ established in Corollary 2.3. It is continuous, since $b_{\iota_{s}}$ is open.

We have $\hat{j}_{0}=i d, \hat{j}_{1}$ maps $\operatorname{St}(\mathfrak{E})_{S}$ onto $\mathrm{U}(\mathfrak{C})_{S}$, and $\left.\hat{j}_{t}\right|_{\mathrm{U}(\mathfrak{C})_{S}}=i d$ for $0 \leqq t \leqq 1$. Hence $\hat{j}_{t}, 0 \leqq t \leqq 1$, is a strong deformation retract homotopy for $\mathrm{U}(\mathbb{E})_{S} \subset \mathrm{St}(\mathbb{E})_{S}$.

8. Grassmann spaces and Stiefel spaces of a Hilbert space. We consider now the maximal $R$-set $\mathfrak{M}=\mathfrak{I}(H)$. In this case $A(\mathfrak{M})=\mathfrak{L}(H)$ and $I(\mathfrak{M})=\mathfrak{L}(H)$, and the theory simplifies essentially. It does not depend on Chapters 4 and 5 at all. Since $\mathrm{GL}(\mathfrak{M})=\mathrm{GL}(H)$ and $\mathrm{U}(\mathfrak{M})=\mathrm{U}(H)$, one has not to take particular care of these groups any more.

This case establishes also a direct connection with the "ordinary theory" of Grassmann and Stiefel spaces of a Hilbert space. In the following we present this ordinary theory independent of the dimension of the Hilbert space. We investigate then the Grassmann and Stiefel spaces of infinite-dimensional Hilbert spaces, and we will obtain a certain complete characterization.

Crucial is the following theorem:

THEOREM 8.1 (SEE [9]). Let $H$ be an infinite-dimensional Hilbert space. The groups $\mathrm{GL}(H)$ and $\mathrm{U}(H)$ are contractible. In particular $\mathrm{GL}(H)$ and $\mathrm{U}(H)$ are connected, and all homotopy groups are trivial.

$$
\pi_{i}(\mathrm{GL}(H))=0 \text { and } \pi_{i}(\mathrm{U}(H))=0 \text { for } i=0,1,2, \ldots
$$

Definition 8.1. Let $\mathfrak{t}$ and $\mathfrak{l}$ be two cardinal numbers with $\mathfrak{t}+\mathfrak{l}=\operatorname{dim}(H)$. The 
Grassmann space $\mathfrak{S S}_{\langle\mathfrak{f}, \mathfrak{l}\rangle}$ of type $\mathfrak{f}, \mathfrak{l}$ of the Hilbert space $H$ is the set $\mathbb{B S}_{\langle\mathfrak{t}, \mathfrak{I \rangle}}=\{S ; S \subset H$ a closed linear subspace with $\operatorname{dim}(S)=\mathfrak{t}$ and $\operatorname{codim}(S)=\mathfrak{l}\}$ with the relative topology induced by the inclusion $\mathbb{S}_{\langle t, l\rangle} \subset \mathfrak{T}(H)$.

If $H$ is finite dimensional, we obtain the ordinary Grassmann manifolds. In this case we can of course dispense with the double index.

If $H$ is a separable infinite dimensional Hilbert space, we denote $\operatorname{dim}(H)=\boldsymbol{\aleph}_{0}$ $=\infty$, and we have the following types of Grassmann spaces

$$
\mathbb{B S}_{\langle n, \infty\rangle}, \quad \mathscr{S S}_{\langle\infty, \infty\rangle}, \quad \mathbb{G S}_{\langle\infty, n\rangle} \text { where } n=0,1,2, \ldots
$$

THEOREM 8.2. The Grassmann spaces $\mathbb{S S}_{\langle\mathfrak{t}, 1\rangle}$ are the path components $\mathbb{E}$ of the space $\mathfrak{T}(H)$. The Grassmann spaces $\mathfrak{B S}_{\langle\mathrm{t}, 1\rangle}$ are therefore connected Banach manifolds (Theorem 6.1), and homogeneous spaces

$$
\dot{S S}_{\langle\mathfrak{t}, \mathfrak{l}\rangle}=\mathrm{GL}(H) / \mathrm{GL}(H)_{S}=\mathrm{U}(H) / \mathrm{U}(H)_{S},
$$

where $S \in \mathbb{S}_{\langle\mathrm{t}, \mathrm{l}\rangle}$.

Proof. Let $S \in \mathbb{S}\langle\{t, 1\rangle$ and let $\mathbb{C}$ be the path component of $\mathfrak{T}(H)$ with $S \in \mathbb{C}$. First we have $\mathbb{C} \subset \mathbb{S}_{\langle\mathfrak{t}, \mathfrak{l}\rangle}$. Namely if $T \in \mathfrak{E}$, then there exists a $\gamma \in \mathrm{GL}(H)$ with $\gamma(S)=T$ and therefore $\operatorname{dim}(T)=\mathfrak{l}$ and $\operatorname{codim}(T)=\mathfrak{l}$. Hence $T \in \mathbb{S}_{\langle\mathfrak{t}, \mathfrak{l}\rangle}$. Now let $T \in \mathbb{S}_{\langle\mathfrak{t}, \mathfrak{}\rangle}$ be given. We show that there is a continuous path in $\mathfrak{T}(H)$ which connects $S$ with $T$. Since $\operatorname{dim}(S)=\operatorname{dim}(T)=\mathfrak{f}$ and $\operatorname{codim}(S)=\operatorname{codim}(T)=\mathfrak{l}$, we can construct a $\gamma \in \mathrm{GL}(H)$ with $\gamma(S)=T$. If $H$ is a finite-dimensional real Hilbert space, we may assume $\gamma \in \mathrm{GL}^{1}(H)$. In all other cases we have $\mathrm{GL}^{1}(H)=\mathrm{GL}(H)$. Therefore there exists a continuous path $\gamma_{t} \in \mathrm{GL}(H), 0 \leqq t \leqq 1$, with $\gamma_{0}=1$ and $\gamma_{1}=\gamma$. Then $\gamma_{t}(S), 0 \leqq t \leqq 1$, is a continuous path in $\mathfrak{T}(H)$ which connects $S$ with $T$ (Lemma 3.4). This shows $T \in \mathfrak{E}$. Hence $\mathfrak{E}=\mathfrak{S S}_{\langle\mathfrak{t}, I\rangle}$.

Corollary 8.1. Let $S \in \mathbb{S}_{\langle t, 1\rangle}$ be fixed. $\mathrm{GL}(H)$ is a principal bundle over $\mathbb{S}_{\langle t, 1\rangle}$ with fibre and structure group $\mathrm{GL}(H)_{S}$, and also $\mathrm{U}(H)$ is a principal bundle over $\mathbb{S S}_{\langle\mathrm{i}, \mathrm{l}\rangle}$ with fibre and structure group $\mathrm{U}(H)_{S}=\mathrm{U}(S) \times \mathrm{U}\left(S^{\perp}\right)$ (Corollaries 5.1 and $\left.5.1^{\prime}\right)$.

THEOREM 8.3. The map $\perp: \mathbb{S}_{\langle\mathrm{r}, \mathrm{l}\rangle} \rightarrow \mathscr{B S}_{\langle 1, \mathrm{t}\rangle}, \perp(S)=S^{\perp}$, is an isometry with respect to the d-metric and to the g-metric.

Proof. Lemma 3.1.

Definition 8.2. Let $\mathfrak{t}$ and $\mathfrak{l}$ be two cardinal numbers with $k+l=\operatorname{dim}(H)$. An orthonormal $\langle\mathfrak{f}, \mathfrak{l}\rangle$-frame $\phi$ in $H$ is an ordered set $\left\{e_{\mathfrak{i}}\right\}$ of power $k$ of pairwise orthogonal vectors of unit length, and if $[\phi]=\overline{\left[\left\{e_{i}\right\}\right]}$ is the closed linear subspace determined by the set $\phi$, then $\operatorname{dim}([\phi])=\mathfrak{f}$ and $\operatorname{codim}([\phi])=\mathfrak{l}$.

A $\langle\mathfrak{t}, \mathfrak{l}\rangle$-frame $\psi$ in $H$ is an ordered set $\left\{v_{i}\right\}$ of power $\mathfrak{t}$ of linearly independent vectors, such that if $[\psi]=\overline{\left[\left\{v_{i}\right\}\right]}$ is the closed linear subspace determined by the set 
$\psi$, we have $\operatorname{dim}([\psi])=\mathfrak{l}$ and $\operatorname{codim}([\psi])=\mathfrak{l}$, and further there exists an orthonormal $\langle\mathfrak{t}, \mathfrak{l}\rangle$-frame $\phi_{0}=\left\{e_{i}\right\}$ such that the linear map

$$
\begin{aligned}
& \alpha_{\left(\phi_{0}, \psi\right)}:\left[\phi_{0}\right] \rightarrow[\psi] \text { determined by } \\
& \alpha_{\left(\phi_{0}, \psi\right)}\left(e_{i}\right)=v_{i}
\end{aligned}
$$

is an isomorphism onto $[\psi]$. This last condition is of course not necessary if $\mathfrak{t}$ is finite.

The Stiefel space $\mathfrak{S}_{\langle\mathfrak{t}, \mathfrak{l}\rangle}$ of type $\mathfrak{f}, \mathfrak{l}$ of the Hilbert space $H$ is the set

$$
\Im_{\langle\mathfrak{t}, \mathfrak{l}\rangle}=\{\psi ; \psi \mathrm{a}\langle\mathfrak{t}, \mathfrak{l}\rangle \text {-frame in } H\} \text {. }
$$

And the orthogonal Stiefel space $\mathfrak{u}_{\langle\mathfrak{t}, \mathfrak{l}\rangle}$ of type $\mathfrak{t}, \mathfrak{l}$ of the Hilbert space $H$ is the set

$$
\mathfrak{u}_{\langle\mathfrak{t}, \mathfrak{l}\rangle}=\{\phi ; \phi \text { an orthogonal }\langle\mathfrak{t}, \mathfrak{l}\rangle \text {-frame in } H\} .
$$

A natural topology on the sets $\mathfrak{S}_{\langle\mathfrak{t}, 1\rangle}$ and $\mathfrak{u}_{\langle\mathfrak{t}, \mathfrak{l}\rangle}$ will be subsequently introduced. Obviously $\mathfrak{u}_{\langle\mathfrak{t}, 1\rangle} \subset \mathfrak{S}_{\langle\mathfrak{t}, 1\rangle}$.

We obtain the ordinary Stiefel manifolds if $H$ is a finite-dimensional vectorspace. In this case we need of course again one index only.

If $H$ is a separable infinite-dimensional Hilbert space, we denote again $\operatorname{dim}(H)=\aleph_{0}=\infty$, and we have the following types of Stiefel spaces

$$
\mathfrak{S}_{\langle n, \infty\rangle}, \quad \boldsymbol{S}_{\langle\infty, \infty\rangle}, \quad \boldsymbol{S}_{\langle\infty, n\rangle},
$$

and the orthogonal Stiefel spaces

$$
\mathfrak{u}_{\langle n, \infty\rangle}, \quad \mathfrak{u}_{\langle\infty, \infty\rangle}, \quad \mathfrak{u}_{\langle\infty, n\rangle} \quad \text { where } n=0,1, \ldots
$$

LEMma 8.1. Let $\phi_{0}=\left\{e_{i}\right\}$ be a fixed orthonormal $\langle\mathfrak{t}, \mathfrak{l}\rangle$-frame of $H$, and let $S=\left[\phi_{0}\right]$. If $H$ is a finite-dimensional real vectorspace, we assume $\mathfrak{t}<\operatorname{dim}(H)$. We consider the following natural map (compare Chapter 7)

$$
\begin{aligned}
& i_{\phi_{0}}: \mathfrak{S}_{\langle t, l\rangle} \rightarrow \operatorname{St}\left(\mathbb{S}_{\langle t, l\rangle}\right)_{S}, \\
& i_{\phi_{0}}(\psi): S \rightarrow H \text { is the linear map uniquely determined } \\
& \text { by } i_{\phi_{0}}(\psi)\left(e_{i}\right)=v_{i} \text { where } \psi=\left\{v_{i}\right\} \in \mathfrak{S}_{\langle i, 1\rangle} \text {. }
\end{aligned}
$$

This map $i_{\phi_{0}}$ defines also

$$
i_{\phi_{0}}: \mathfrak{u}_{\langle\mathfrak{z}, 1\rangle} \rightarrow \mathrm{U}\left(\mathfrak{S}_{\langle\mathfrak{f}, \mathfrak{l}\rangle}\right)_{S} .
$$

The map $i_{\phi_{0}}$ is bijective in both cases.

Definition 8.3. Let $S \in \mathcal{G}_{\langle t, 1\rangle}$. Then we identify $\mathfrak{S}_{\langle\mathfrak{t}, 1\rangle}$ with $\operatorname{St}\left(\mathcal{S}_{\langle t, 1\rangle}\right)_{S}$, and $\mathfrak{u}_{\langle\mathfrak{t}, 1\rangle}$ with $\mathrm{U}\left(\mathbb{S}_{\langle\mathfrak{t}, 1\rangle}\right)_{S}$. If $H$ is a finite-dimensional real vectorspace and $\mathfrak{f}=\operatorname{dim} H$, then we identify $\mathfrak{S}_{\langle\operatorname{dim}(H), 0\rangle}$ with $\mathrm{GL}(H)$ and $\mathfrak{u}_{\langle\operatorname{dim}(H), 0\rangle}$ with $\mathrm{U}(H)$.

THEOREM 8.4. The Stiefel spaces $\mathfrak{}_{\langle\mathrm{i}, 1\rangle}$ and $\mathfrak{u}_{\langle\mathrm{t}, 1\rangle}$ are Banach manifolds. They are connected except for $H$ a finite-dimensional real vectorspace and $\mathfrak{t}=\operatorname{dim} H$. 
(In this case $\mathfrak{S}_{\langle\operatorname{dim}(H), 0\rangle}=\mathrm{GL}(H)$ and $\mathfrak{u}_{\langle\mathrm{dim}(H), 0\rangle}=\mathrm{U}(H)$ have exactly two path components.) The Stiefel spaces are also homogeneous spaces. We have

$$
\mathfrak{S}_{\langle\mathfrak{t}, \mathfrak{l}\rangle}=\mathrm{GL}(H) / \mathrm{GL}(H)_{\{S\rangle} \text { and } \mathfrak{u}_{\langle\mathfrak{t}, \mathfrak{l}\rangle}=\mathrm{U}(H) / \mathrm{U}(H)_{\{S\rangle},
$$

where $S \in \mathfrak{S}_{\langle\mathfrak{t}, \mathrm{l}\rangle}$. Finally $\mathfrak{u}_{\langle\mathfrak{t}, 1\rangle} \subset \mathfrak{S}_{\langle\mathfrak{t}, 1\rangle}$ is a strong deformation retract of the space $\mathfrak{S}_{\langle\mathfrak{t}, \mathfrak{l}\rangle}$. In particular $\mathfrak{u}_{\langle\mathfrak{t}, \mathfrak{l}\rangle}$ and $\mathfrak{S}_{\langle\mathfrak{t}, \mathrm{l}\rangle}$ are of the same homotopy type.

Corollary 8.2. Let $S \in \mathbb{S}_{\langle\mathfrak{t}, 1\rangle}$. Then $\mathrm{GL}(H)$ is a principal fibre bundle over $\mathfrak{S}_{\langle\mathfrak{t}, 1\rangle}=\mathrm{GL}(H) / \mathrm{GL}(H)_{\langle S\rangle}$ with fibre and structure group $\mathrm{GL}(H)_{\{S\rangle}$, which acts on the fibres by left translations. And $\mathrm{U}(H)$ is a principal fibre bundle over $\mathfrak{u}_{\langle\mathrm{t}, 1\rangle}$ $=\mathrm{U}(H) / \mathrm{U}(H)_{\{S\}}$ with fibre and structure group $\mathrm{U}(H)_{\{S\}}=\mathrm{U}\left(S^{\perp}\right)$, which acts on the fibres by left translations.

THEOREM 8.5. Let $H$ be an infinite-dimensional Hilbert space. The projection map $p: \mathrm{U}(H) \rightarrow \mathfrak{U}_{\langle\mathrm{t}, 1\rangle}=\mathrm{U}(H) / \mathrm{U}(H)_{\{S\}}$, where $S \in \mathbb{S}_{\langle\mathfrak{t}, 1\rangle}$, determines a universal classifying principal bundle for the group $\mathrm{U}\left(S^{\perp}\right)$. (For the definition of universal classifying principal bundles see, for example, $[14, p .100].) \mathfrak{u}_{\langle\mathfrak{q}, \mid\rangle}$ is therefore a universal classifying space $B_{\mathrm{U}\left(H^{\prime}\right)}$ for the group $\mathrm{U}\left(H^{\prime}\right)$, where $H^{\prime}$ is a Hilbert space with $\operatorname{dim}\left(H^{\prime}\right)=\mathfrak{l}$. In particular $\mathfrak{u}_{\langle\mathfrak{f}, n\rangle}$ is a universal classifying space $B_{U(n)}$ for the orthogonal group $\mathrm{U}(n)$ of the $n$-dimensional vectorspace, $n=0,1, \ldots$

Proof. By Theorem 8.1, $\pi_{i}(U(H))=0, i=0,1, \ldots$, and the theorem on p. 102 in [14].

COROllary 8.3. Let $H$ be an infinite-dimensional Hilbert space. The homotopy groups of the Stiefel spaces $\mathfrak{u}_{\langle\mathfrak{t}, 1\rangle}$ and $\mathfrak{S}_{\langle\mathfrak{t}, 1\rangle}$ are

$$
\begin{aligned}
& \pi_{i}\left(\mathfrak{U}_{\langle\mathfrak{t}, \mathfrak{l}\rangle}\right)=\pi_{i}\left(\Im_{\langle\mathfrak{t}, \mathfrak{l}\rangle}\right)=0, \quad i=0,1,2, \ldots, \text { if } \mathfrak{l} \text { is not an integer, } \\
& \pi_{0}\left(\mathfrak{U}_{\langle\mathfrak{t}, n\rangle}\right)=\pi_{0}\left(\Im_{\langle\mathfrak{t}, n\rangle}\right)=0, \text { for } n=0,1,2, \ldots, \\
& \pi_{i}\left(\mathfrak{U}_{\langle\mathfrak{t}, n\rangle}\right)=\pi_{i}\left(\Im_{\langle\mathfrak{t}, n\rangle}\right)=\pi_{i-1}(\mathrm{U}(n)), \quad i=1,2, \ldots, \text { for } n=0,1, \ldots
\end{aligned}
$$

And the singular homology and cohomology with coefficients in the ring of integers $Z$ is

$$
\begin{aligned}
& H\left(\mathfrak{U}_{\langle\mathfrak{t}, \mathfrak{l}\rangle} ; Z\right)=H\left(\Im_{\langle\mathfrak{l}, \mathfrak{l}\rangle} ; Z\right)=0, \text { if } \mathfrak{l} \text { is not an integer, } \\
& H\left(\mathfrak{U}_{\langle\mathfrak{l}, n\rangle} ; Z\right)=H\left(\Im_{\langle\mathfrak{t}, n\rangle} ; Z\right)=H\left(B_{\mathrm{U}(n)} ; Z\right) \text { for } n=0,1,2, \ldots
\end{aligned}
$$

For a description of the cohomology ring $H^{*}\left(B_{\mathrm{U}(n)} ; Z\right)$ see, for example, [2].

Proof. By Theorem $8.4, \mathfrak{u}_{\langle t, 1\rangle}$ and $\mathfrak{S}_{\langle t, 1\rangle}$ are of the same homotopy type. The exact sequence for the homotopy groups of the principal bundle $p: \mathrm{U}(H) \rightarrow$ $\mathrm{U}(H) / \mathrm{U}(H)_{\{S\rangle}$ gives immediately the homotopy groups of the space $\mathfrak{u}_{\langle\mathrm{t}, \mathrm{l}\rangle}$. The vanishing of the singular homology groups and therefore of the singular cohomol- 
ogy groups in the first case follows from the Hurewicz isomorphism theorem which relates homotopy groups and singular homology groups (see, for example, $[14$, p. 79$])$.

COROLlaRY 8.4. In particular let $H$ be a separable infinite-dimensional Hilbert space. Then the homotopy groups of the Stiefel spaces are

$$
\begin{aligned}
& \pi_{i}\left(\Im_{\langle n, \infty\rangle}\right)=\pi_{i}\left(\mathfrak{u}_{\langle n, \infty\rangle}\right)=0, \quad i=0,1,2, \ldots, \text { for } n=0,1,2, \ldots, \\
& \pi_{i}\left(\Im_{\langle\infty, \infty\rangle}\right)=\pi_{i}\left(\mathfrak{U}_{\langle\infty, \infty\rangle}\right)=0, \quad i=0,1,2, \ldots, \\
& \pi_{0}\left(\Im_{\langle\infty, n\rangle}\right)=\pi_{0}\left(\mathfrak{U}_{\langle\infty, n\rangle}\right)=0, \text { for } n=0,1,2, \ldots, \\
& \pi_{i}\left(\Im_{\langle\infty, n\rangle}\right)=\pi_{i}\left(\mathfrak{U}_{\langle\infty, n\rangle}\right)=\pi_{i-1}(U(n)), \quad i=1,2, \ldots, \text { for } n=0,1,2, \ldots
\end{aligned}
$$

THEOREM 8.6. Let $S \in \mathbb{B S}_{\langle\mathrm{t}, 1\rangle}$. The projection map

$$
q: \mathfrak{\Im}_{\langle\mathfrak{t}, 1\rangle}=\mathrm{GL}(H) / \mathrm{GL}(H)_{\{S\}} \rightarrow \mathfrak{S S}_{\langle\mathfrak{t}, \mathfrak{l}\rangle}=\mathrm{GL}(H) / \mathrm{GL}(H)_{S}
$$

defines a principal bundle over $\mathbb{S}_{\langle\mathrm{f}, 1\rangle}$ with fibre and structure group $\mathrm{GL}(S)$, which acts on the fibres by left translations. And the projection map

$$
q: \mathfrak{u}_{\langle\mathfrak{t}, \mathfrak{l}\rangle}=\mathrm{U}(H) / \mathrm{U}(H)_{\langle S\rangle} \rightarrow \mathbb{S}_{\langle\mathfrak{t}, \mathfrak{l}\rangle}=\mathrm{U}(H) / \mathrm{U}(H)_{S}
$$

defines a principal bundle over $\mathfrak{B S}_{\langle\mathfrak{t}, \mathrm{l}\rangle}$ with fibre and structure group $\mathrm{U}(S)$, which acts on the fibres by left translations.

CoROllaRY 8.5. Let $H$ be an infinite-dimensional Hilbert space. Suppose $\mathfrak{l}$ is not an integer. Then the projection $q: \mathfrak{S}_{\langle\mathfrak{f}, l\rangle} \rightarrow \mathbb{S}_{\langle\mathfrak{t}, l\rangle}$ determines a universal classifying principal bundle for the group $\mathrm{GL}\left(H^{\prime}\right)$, and the projection $q: \mathfrak{u}_{\langle\mathrm{t}, \mathrm{l}\rangle} \rightarrow \mathfrak{S S}_{\langle\mathrm{t}, \mathrm{I}\rangle} a$ universal classifying principal bundle for the group $\mathrm{U}\left(H^{\prime}\right)$, where $H^{\prime}$ is a Hilbert space with $\operatorname{dim}\left(H^{\prime}\right)=\mathfrak{t} \mathbb{S}_{\langle\mathfrak{t}, 1\rangle}$ is therefore a universal classifying space $B_{\mathrm{GL}\left(H^{\prime}\right)}$ and $B_{\mathrm{U}\left(H^{\prime}\right)}$ for the groups $\mathrm{GL}\left(H^{\prime}\right)$ and $\mathrm{U}\left(H^{\prime}\right)$. In particular $\mathbb{S S}_{\langle n, 1\rangle}$ is a universal classifying space $B_{\mathrm{GL}(n)}$ and $B_{\mathrm{U}(n)}$ for the groups $\mathrm{GL}(n)$ and $\mathrm{U}(n)$ of the $n$-dimensional vectorspace.

COROLlaRY 8.6. Let $H$ be an infinite-dimensional Hilbert space. The homotopy groups of the Grassmann spaces ${ }^{(S)}\langle\mathfrak{i}, 1\rangle$ are

$$
\begin{aligned}
& \pi_{i}\left(\mathscr{S}_{\langle\mathfrak{t}, \mathfrak{l}\rangle}\right)=0, \text { if neither } \mathfrak{t} \text { nor } \mathfrak{l} \text { is an integer, } \\
& \pi_{0}\left(\mathscr{S}_{\langle n, \mathfrak{l}\rangle}\right)=\pi_{0}\left(\mathbb{S}_{\langle\mathfrak{t}, n\rangle}\right)=0, \text { for } n=0,1,2, \ldots, \\
& \pi_{i}\left(\mathscr{S}_{\langle n, 1\rangle}\right)=\pi_{i}\left(\mathfrak{S}_{\langle\mathfrak{t}, n\rangle}\right)=\pi_{i-1}(\mathrm{U}(n)), \quad i=1,2, \ldots, \text { for } n=0,1,2, \ldots
\end{aligned}
$$

And the singular homology and cohomology with coefficients in the ring of integers $Z$ is

$$
\begin{aligned}
& H\left(\mathscr{S}_{\langle\mathfrak{t}, \mathfrak{l}\rangle} ; Z\right)=0, \quad \text { if neither } \mathfrak{t} \text { nor } \mathfrak{l} \text { is an integer, } \\
& H\left(\mathcal{S}_{\langle n, \mathfrak{l}\rangle} ; Z\right)=H\left(\mathfrak{S}_{\langle\mathfrak{l}, n\rangle} ; Z\right)=H\left(B_{\mathrm{U}(n)} ; Z\right), \text { for } n=0,1,2, \ldots
\end{aligned}
$$


COROLlARY 8.7. Let $H$ be a separable infinite-dimensional Hilbert space. Then the homotopy groups of the Grassmann spaces are

$$
\begin{aligned}
\pi_{i}\left(\mathbb{S}_{\langle\infty, \infty\rangle}\right) & =0, \quad i=0,1,2, \ldots, \\
\pi_{0}\left(\mathbb{S}_{\langle n, \infty\rangle}\right) & =\pi_{0}\left(\mathbb{S}_{\langle\infty, n\rangle}\right)=0, \text { for } n=0,1,2, \ldots, \\
\pi_{i}\left(\mathbb{S}_{\langle n, \infty\rangle}\right) & =\pi_{i}\left(\mathcal{S}_{\langle\infty, n\rangle}\right)=\pi_{i-1}(\mathrm{U}(n)), \quad i=1,2, \ldots, \text { for } n=0,1,2, \ldots
\end{aligned}
$$

REMARKS. It is easy to associate with the various classifying spaces of type $B_{\mathrm{GL}(n)}$ and $B_{\mathrm{U}(n)}$ in this chapter in a natural way universal classifying vector bundles of dimension $n$. Since all spaces which occur in this chapter are Banach manifolds, it follows from Theorem 15 of [13] that all maps in this chapter which induce isomorphisms for the homotopy groups are homotopy equivalences. In particular all spaces with vanishing homotopy groups are contractible.

9. The general case. There appear various examples of maximal $R$-sets in analysis (see [4]). In a subsequent publication we intend to compute the homotopy type of the path components and of the associated groups of some of these examples. Theorem 5.5, Corollaries 6.2 and $6.2_{q}$, and Theorem 9.1 imply the following theorem, which can be used for computations:

THEOREM 9.1. Let $\mathfrak{M}$ be a maximal $R$-set, and let $\mathbb{E} \in \pi_{0}(\mathfrak{M})$ be a path component of $\mathfrak{M}$ such that there is a $S \in \mathfrak{C}$ with $\operatorname{codim}(S)$ infinite. Suppose also that $\mathrm{U}\left(\left.\mathfrak{M}\right|_{S}\right)$ and $\mathrm{U}_{q}\left(I\left(\left.\mathfrak{M}\right|_{S}\right)\right)$ are connected. Then we have the exact sequences of homotopy groups

$$
\begin{aligned}
\cdots \rightarrow \pi_{n+1}(\mathfrak{E}) \rightarrow \pi_{n}\left(\mathrm{U}\left(\left.\mathfrak{M}\right|_{S}\right)\right) & \rightarrow \pi_{n}\left(\mathrm{U}^{1}(\mathfrak{M})\right) \rightarrow \pi_{n}(\mathfrak{E}) \rightarrow \cdots \\
\cdots \rightarrow \pi_{n+1}(\mathfrak{E}) \rightarrow \pi_{n}\left(\mathrm{U}_{q}\left(I\left(\left.\mathfrak{M}\right|_{S}\right)\right)\right) & \rightarrow \pi_{n}\left(\mathrm{U}_{q}^{1}(I(\mathfrak{M}))\right) \rightarrow \pi_{n}(\mathfrak{E}) \rightarrow \cdots .
\end{aligned}
$$

\section{REFERENCES}

1. N. I. Ahiezer and I. M. Glazman, Theorie der linearen Operatoren im Hilbert-Raum, Akademie-Verlag, Berlin, 1954.

2. A. Borel, Sur la cohomologie des espaces fibrés principaux et des espaces homogènes de groupes de Lie compacts, Ann. of Math. 57 (1953), 115-207.

3. H. O. Cordes and J. P. Labrousse, The invariance of the index in the metric space of closed operators, J. Math. Mech. 12 (1963), 693-720.

4. H. O. Cordes, On a class of $C^{*}$-algebras, Math. Ann. (to appear).

5. - On a generalized Fredholm theory, Math. Ann. (to appear).

6. - Uber eine nichtalgebraische Charakterisierung von I-Fredholm-Operatoren, Math. Ann. (to appear).

7. C. Z. Gohberg and M. G. Krein, The basic propositions on defect numbers, root numbers and indices of linear operators, Amer. Math. Soc. Transl. (2) 13 (1960), 185-264.

8. T. Kato, Perturbation theory for nullity, deficiency and other quantities of linear operators, J. Analyse Math. 6 (1958), 261-322.

9. N. H. Kuiper, The homotopy type of the unitary group of Hilbert space, Topology 3 (1965), 19-30. 
10. S. Lang, Introduction to differentiable manifolds, Interscience, New York, 1962.

11. L. H. Loomis, An introduction to abstract harmonic analysis, Van Nostrand, New York, 1953.

12. B. Sz. Nagy, Spektraldarstellung linearer Transformationen des Hilbertschen Raumes, Ergebnisse der Mathematik, Springer, Berlin, 1942.

13. R. S. Palais, Homotopy theory of infinite-dimensional manifolds, Topology 5 (1966), 1-16.

14. N. Steenrod, The topology of fibre bundles, Princeton Univ. Press, Princeton, N. J., 1951.

UNIVERSITY OF BRITISH COLUMBIA,

VANCOUver, British Columbia, Canada 